\title{
The Total Synthesis of Psymberin
}

Xianhai Huang,* Ning Shao, Anandan Palani, Robert Aslanian, and Alexei Buevich Department of Chemical Research, Schering-Plough Research Institute, Kenilworth, NJ 07033

Corresponding Author: xianhai.huang@ spcorp.com

\section{Supporting Information}

\section{Contents:}

1. Experimental Procedures (page S1)

2. Spectrum comparison of synthetic psymberin and natural psymberin (page S22)

3. Structure determination of epi-1 (page S25)

Analytical methods: All reactions were carried out under a nitrogen atmosphere with dry solvents under anhydrous conditions, unless otherwise noted. Reagents were purchased at the highest commercial quality and used without further purification, unless otherwise stated. Flash chromatography was carried out with EM science Silica gel 60 (neutral, 230-400 mesh). ${ }^{1} \mathrm{H}$ NMR and ${ }^{13} \mathrm{C}$ NMR spectra were recorded on a Bruker Avance 500 NMR Spectrometer with chemical shifts reported in ppm relative to the residual deuterated solvent. LCMS was recorded on Applied Biosystem API-150.

\section{Experimental Procedures:}

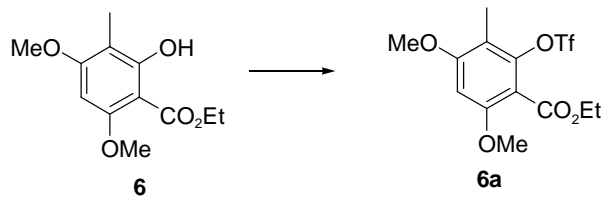

To a solution of compound 6 (22g, $91.4 \mathrm{mmol}$, prepared from 2,4,6-trimethoxytoluene in two step in $46 \%$ yield according to literature procedure: Solladie, G.; Gehrold, N.; Maignan, J. Tetrahedron: Asymmetry 1999, 10, 2739.) in DCM (200 ml) was added pyridine $(22 \mathrm{ml})$ at $0{ }^{\circ} \mathrm{C} . \mathrm{Tf}_{2} \mathrm{O}(23 \mathrm{ml}, 137 \mathrm{mmol})$ was added dropwise and the reaction mixture was allowed to stir at $0{ }^{\circ} \mathrm{C}$ overnight before it was diluted with DCM $(200 \mathrm{ml})$ and washed with aq. $\mathrm{NH}_{4} \mathrm{Cl}(200 \mathrm{ml}), \mathrm{NaHCO}_{3}$ and brine sequentially. After being dried over $\mathrm{Na}_{2} \mathrm{SO}_{4}$ and concentration, the residue was purified by FC (silica gel, EtOAc/Hexane, 1/10) to give phenol triflate 6a (34 g, $91.3 \mathrm{mmol}, 99 \%) .{ }^{1} \mathrm{HNMR}$ 
$\left(\mathrm{CDCl}_{3}\right) \delta 6.45(\mathrm{~s}, 1 \mathrm{H}), 4.34(\mathrm{q}, J=7.0 \mathrm{~Hz}, 2 \mathrm{H}), 3.89(\mathrm{~s}, 3 \mathrm{H}), 3.87(\mathrm{~s}, 3 \mathrm{H}), 2.15(\mathrm{~s}, 3 \mathrm{H})$, $1.36(\mathrm{t}, J=7.0 \mathrm{~Hz}, 2 \mathrm{H})$; MS (ES) calculated for $\mathrm{C}_{12} \mathrm{H}_{12} \mathrm{~F}_{3} \mathrm{O}_{6} \mathrm{~S}[\mathrm{M}+\mathrm{H}]^{+} 341.03$, found 341.03 .

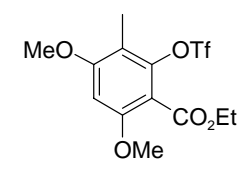

6 a

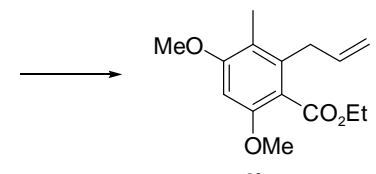

$6 \mathrm{~b}$

To a solution of compound $\mathbf{6 a}(34 \mathrm{~g}, 91.3 \mathrm{mmol})$ in degassed anhydrous THF (500 ml) were added $\mathrm{Pd}\left(\mathrm{PPh}_{3}\right)_{4}(4 \mathrm{~g}, 3.5 \mathrm{mmol}), \mathrm{LiCl}(12 \mathrm{~g}, 274 \mathrm{mmol})$, and allyltributyltin (43 $\mathrm{ml}, 137 \mathrm{mmol}$ ). The reaction mixture was heated to reflux under $\mathrm{N}_{2}$ and allowed to stir at reflux overnight. After the reaction was cooled to room temperature, it was diluted with EtOAc $(300 \mathrm{ml})$. The reaction mixture was filtered through a celite pad, washed with EtOAc and the organic solution combined. After washing with $10 \%$ aq. $\mathrm{NH}_{4} \mathrm{OH}(200$ $\mathrm{ml}$ ), brine $(200 \mathrm{ml})$, and dried over $\mathrm{MgSO}_{4}$, it was concentrated and purified by FC (silica gel, EtOAc/Hexane 1/20 to 1/10) to afford compound $\mathbf{6 b}(22.8 \mathrm{~g}, 95 \%) .{ }^{1} \mathrm{HNMR}\left(\mathrm{CDCl}_{3}\right)$ $\delta 6.37(\mathrm{~s}, 1 \mathrm{H}), 5.89-5.82(\mathrm{~m}, 1 \mathrm{H}), 5.02-4.93(\mathrm{~m}, 2 \mathrm{H}), 4.35(\mathrm{q}, J=7.0 \mathrm{~Hz}, 2 \mathrm{H}), 3.84(\mathrm{~s}$, $3 \mathrm{H}), 3.82(\mathrm{~s}, 3 \mathrm{H}), 3.35(\mathrm{dd}, J=1.5,1.5 \mathrm{~Hz}, 1 \mathrm{H}), 3.34(\mathrm{dd}, J=1.5,1.5 \mathrm{~Hz}, 1 \mathrm{H}), 2.08$ (s, $3 \mathrm{H}), 1.35(\mathrm{t}, J=7.0 \mathrm{~Hz}, 3 \mathrm{H}) ;{ }^{13} \mathrm{C} \mathrm{NMR}\left(\mathrm{CDCl}_{3}\right) \delta 159.4,155.7,137.0,135.7,118.0$, 117.4, 115.9, 93.9, 61.2, 56.3, 55.9, 35.2, 14.5, 11.0; MS (ES) calculated for $\mathrm{C}_{15} \mathrm{H}_{21} \mathrm{O}_{4}$ $[\mathrm{M}+\mathrm{H}]^{+}$265.14, found 265.32 .

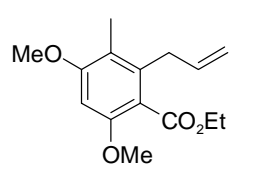

$6 b$

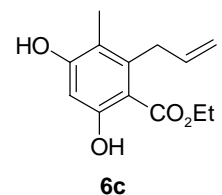

$6 c$

To a solution of compound $\mathbf{6 b}(12.26 \mathrm{~g}, 46 \mathrm{mmol})$ in anhydrous DCM (150 ml) was added $\mathrm{BBr}_{3}(200 \mathrm{ml}, 1.0 \mathrm{M}$ in $\mathrm{DCM}, 200 \mathrm{mmol})$ at $-78{ }^{\circ} \mathrm{C}$. The reaction mixture was allowed to stir at $-78{ }^{\circ} \mathrm{C}$ for 30 minutes and the acetone ice bath was changed to ice bath. The reaction was further stirred at $0{ }^{\circ} \mathrm{C}$ for 4 hours, and additional 45 minutes at $\mathrm{rt}$. The reaction was cooled to $0{ }^{\circ} \mathrm{C}$ before water $(100 \mathrm{ml})$ was added. It was extracted with EtOAc $(300 \mathrm{ml})$ and washed with water. Organic layer was dried and concentrated. The residue was purified by FC (acetone/Hexane 1/10) to give the phenol 6c $(9.2 \mathrm{~g}, 39 \mathrm{mmol}$, $85 \%)$ as a brown solid. ${ }^{1} \mathrm{HNMR}\left(\mathrm{CDCl}_{3}\right) \delta 11.5(\mathrm{~s}, 1 \mathrm{H}), 6.34(\mathrm{~s}, 1 \mathrm{H}), 5.97-5.89(\mathrm{~m}, 1 \mathrm{H})$, $5.84(\mathrm{~s}, 1 \mathrm{H}), 5.03-4.99(\mathrm{~m}, 1 \mathrm{H}), 4.90-4.86(\mathrm{~m}, 1 \mathrm{H}), 4.38(\mathrm{q}, J=7.5 \mathrm{~Hz}, 2 \mathrm{H}), 3.72$ (dd, $J=$ 
$1.5,1.5 \mathrm{~Hz}, 1 \mathrm{H}), 3.71(\mathrm{dd}, J=1.5,1.5 \mathrm{~Hz}, 1 \mathrm{H}), 2.11(\mathrm{~s}, 3 \mathrm{H}), 1.38(\mathrm{t}, J=7.5 \mathrm{~Hz}, 3 \mathrm{H}) ;{ }^{13} \mathrm{C}$ NMR $\left(\mathrm{CDCl}_{3}\right) \delta 171.7,162.1,159.2,142.3,136.6,115.1,101.8,61.7,35.8,14.3,11.1$.

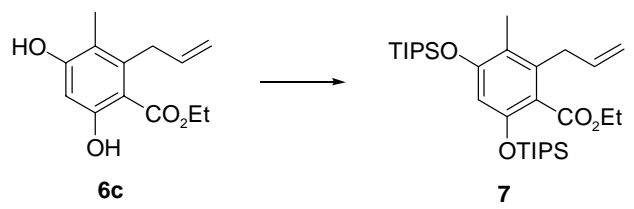

To a solution of compound $\mathbf{6 c}(7 \mathrm{~g}, 29.6 \mathrm{mmol})$ in DCM (150 ml) was added 2, 6-lutidine $(14 \mathrm{ml}, 120 \mathrm{mmol})$ at $0{ }^{\circ} \mathrm{C}$. After stirring for 5 minutes at $0{ }^{\circ} \mathrm{C}$, TIPSOTf $(24 \mathrm{ml}, 90$ mmol) was added dropwise. The reaction mixture was allowed to stir at room temperature for 2 hours. After the reaction was complete as judged by TLC, it was diluted with EtOAc $(300 \mathrm{ml})$. The organic layer was washed with $\mathrm{NaHCO}_{3}$, dried over $\mathrm{Na}_{2} \mathrm{SO}_{4}$ and concentrated. The crude was purified by FC (EtOAc/Hexane 1/10) to give product $7(15.4 \mathrm{~g}, 95 \%)$ as an oil: ${ }^{1} \mathrm{HNMR}\left(\mathrm{CDCl}_{3}\right) \delta 6.25(\mathrm{~s}, 1 \mathrm{H}), 5.90-5.82(\mathrm{~m}, 1 \mathrm{H})$, 5.01-4.91 (m, 2H), 4.28 (q, $J=7.5 \mathrm{~Hz}, 2 \mathrm{H}), 3.31$ (dd, $J=1.5,1.5 \mathrm{~Hz}, 1 \mathrm{H}), 3.30$ (dd, $J=$ 1.5, $1.5 \mathrm{~Hz}, 1 \mathrm{H}), 2.09$ (s, 3H), $1.32(\mathrm{t}, J=7.5 \mathrm{~Hz}, 3 \mathrm{H}), 1.30-1.21(\mathrm{~m}, 6 \mathrm{H}), 1.11-1.05(\mathrm{~m}$, $36 \mathrm{H}) ;{ }^{13} \mathrm{C} \mathrm{NMR}\left(\mathrm{CDCl}_{3}\right) \delta 169.3,155.4,151.1,136.8,135.9,120.7,120.4,115.6,107.3$, $61.0,35.5,18.2,18.1,14.4,13.4,13.2,11.9$; MS (ES) calculated for $\mathrm{C}_{31} \mathrm{H}_{57} \mathrm{O}_{4} \mathrm{Si}_{2}[\mathrm{M}+$ $\mathrm{H}]^{+}$549.38, found 549.36 .

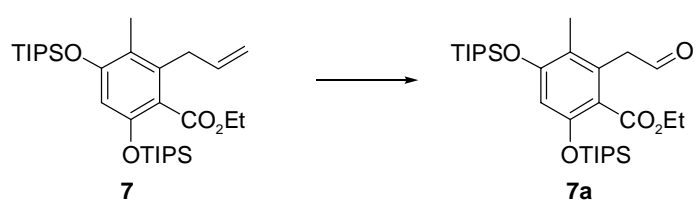

To a solution of $7(15.4 \mathrm{~g}, 28.4 \mathrm{mmol})$ in THF/ $\mathrm{H}_{2} \mathrm{O}(100 / 20 \mathrm{ml})$ were added NMO (7 g, $60 \mathrm{mmol})$ and $\mathrm{OsO}_{4}(18 \mathrm{ml}, 2.5 \% \mathrm{wt}$ in $t-\mathrm{BuOH}, 1.5 \mathrm{mmol})$ at $0{ }^{\circ} \mathrm{C}$. After stirring at ambient temperature for 12 hours, $10 \% \mathrm{Na}_{2} \mathrm{~S}_{2} \mathrm{O}_{3}$ solution $(50 \mathrm{ml})$ was added and the mixture was further stirred for 5 hours. It was then extracted with EtOAc $(250 \mathrm{ml})$ and the combined organic extracts were washed with water and dried over $\mathrm{MgSO}_{4}$. After concentration, the residue obtained was filtered through a short silica gel plug eluted with EtOAc. Removal of the solvent gave the crude diol. $\mathrm{NaIO}_{4}(19.2 \mathrm{~g}, 90 \mathrm{mmol})$ was added to a solution of the crude diol in $90 \% \mathrm{MeOH} / \mathrm{H}_{2} \mathrm{O}(120 \mathrm{ml})$. After stirring at room temperature for 1 hour, EtOAc (300 ml) was added and the reaction mixture was washed 
with water and dried over $\mathrm{MgSO}_{4}$. After concentration, the residue was purified by $\mathrm{FC}$ (silica gel) to give the aldehyde $7 \mathbf{a}(13.7 \mathrm{~g}, 88 \%)$ as a syrup: ${ }^{1} \mathrm{HNMR}\left(\mathrm{CDCl}_{3}\right) \delta 9.60(\mathrm{t}, J$ $=2.5 \mathrm{~Hz}, 1 \mathrm{H}), 6.33(\mathrm{~s}, 1 \mathrm{H}), 4.29$ (q, $J=7.5 \mathrm{~Hz}, 2 \mathrm{H}), 3.59$ (d, $J=2.0 \mathrm{~Hz}, 2 \mathrm{H}), 2.07$ (s, $3 \mathrm{H}), 1.32(\mathrm{t}, J=7.0 \mathrm{~Hz}, 3 \mathrm{H}), 1.29-1.22(\mathrm{~m}, 6 \mathrm{H}), 1.11-1.05(\mathrm{~m}, 36 \mathrm{H}) ;{ }^{13} \mathrm{C} \mathrm{NMR}\left(\mathrm{CDCl}_{3}\right)$ $\delta 199.4,168.8,156.0,151.9,130.3,121.3,120.9,108.6,61.4,46.3,18.2,18.1,17.9,14.4$, $13.4,13.2,12.5$.

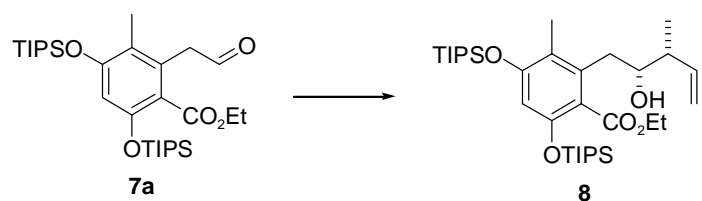

To a suspension of predried $\mathrm{KO}^{t} \mathrm{Bu}(6.5 \mathrm{~g}, 58 \mathrm{mmol})$ in $\mathrm{THF}(100 \mathrm{ml})$ at $-78{ }^{\circ} \mathrm{C}$ was added cis-2-butene (15 ml). $n$-Butyl lithium (36 ml, $1.6 \mathrm{M}$ in Hexane, $58 \mathrm{mmol}$ ) was added slowly over a period of 10 minutes at $-78{ }^{\circ} \mathrm{C}$. The resulting yellow reaction mixture was warmed to $-46{ }^{\circ} \mathrm{C}$ for 30 minutes and then cooled to $-78{ }^{\circ} \mathrm{C}$ and (-)methoxydiisopinocamphenyl borane $(18.3 \mathrm{~g}, 58 \mathrm{mmol})$ in THF $(30 \mathrm{ml})$ was added dropwise. The reaction mixture was stirred for 30 minutes at $-78{ }^{\circ} \mathrm{C}$. Freshly distilled $\mathrm{BF}_{3} \mathrm{OEt}(7.4 \mathrm{ml}, 58 \mathrm{mmol})$ was added to the reaction mixture at $-78{ }^{\circ} \mathrm{C}$ followed by addition of aldehyde $7 \mathbf{a}(8 \mathrm{~g}, 14.8 \mathrm{mmol})$. The reaction mixture was stirred at $-78{ }^{\circ} \mathrm{C}$ for 6 hours. The reaction mixture was poured into $\mathrm{pH} 7$ buffer $(100 \mathrm{ml})$ at $-78{ }^{\circ} \mathrm{C}$ and then 30 $\% \mathrm{H}_{2} \mathrm{O}_{2}(20 \mathrm{ml})$ was added before it was put in an ice bath and further stirred from $0{ }^{\circ} \mathrm{C}$ to room temperature overnight. The reaction mixture was extracted with EtOAc. The organic layers were combined, washed with saturated $\mathrm{Na}_{2} \mathrm{SO}_{3}$ and brine, dried over $\mathrm{Na}_{2} \mathrm{SO}_{4}$, filtered and concentrated under reduced pressure. The resulting crude oil was purified by FC (silica gel; hexane to $2 \%$ EtOAc/hexane) to afford $\mathbf{8}(8 \mathrm{~g}, 89 \%)$ as a single diastereomer by NMR. Enantioselectivity was determined by HPLC using a Chiral OD-H column with $1 \%$ isopropanol in hexane and a flow rate of $1 \mathrm{ml} / \mathrm{min}$. $[\alpha]^{20} \mathrm{D}=+36.5(\mathrm{c}=$ 0.1, DCM). ${ }^{1} \mathrm{HNMR}\left(\mathrm{CDCl}_{3}\right) \delta 6.25(\mathrm{~s}, 1 \mathrm{H}), 5.92-5.85(\mathrm{~m}, 1 \mathrm{H}), 5.12-5.05(\mathrm{~m}, 2 \mathrm{H}), 4.43-$ $4.36(\mathrm{~m}, 1 \mathrm{H}), 4.26-4.20(\mathrm{~m}, 1 \mathrm{H}), 3.63(\mathrm{br}, 1 \mathrm{H}), 3.17$ (d, $J=6.0 \mathrm{~Hz}, 1 \mathrm{H}), 2.93$ (dd, $J=$ 2.5, 14.0 Hz, 1H), 2.41 (dd, $J=11.0,14.5 \mathrm{~Hz}, 1 \mathrm{H}), 2.34-2.31(\mathrm{~m}, 1 \mathrm{H}), 2.12(\mathrm{~s}, 3 \mathrm{H}), 1.35$ $(\mathrm{t}, J=7.0 \mathrm{~Hz}, 3 \mathrm{H}), 1.31-1.18(\mathrm{~m}, 6 \mathrm{H}), 1.13-1.06(\mathrm{~m}, 36 \mathrm{H}) ;{ }^{13} \mathrm{C} \mathrm{NMR}\left(\mathrm{CDCl}_{3}\right) \delta 170.9$, 156.0, 151.4, 141.2, 137.1, 120.6, 120.4, 115.0, 107.4, 74.3, 61.8, 45.3, 36.1, 18.2 , 18.1, 
15.6, 14.4, 13.4, 13.3, 12.5; MS (ES) calculated for $\mathrm{C}_{34} \mathrm{H}_{63} \mathrm{O}_{5} \mathrm{Si}_{2}[\mathrm{M}+\mathrm{H}]^{+}: 607.42$, found 607.38; $\mathrm{C}_{34} \mathrm{H}_{62} \mathrm{NaO}_{5} \mathrm{Si}_{2}[\mathrm{M}+\mathrm{Na}]^{+}: 629.40$, found 629.34 .

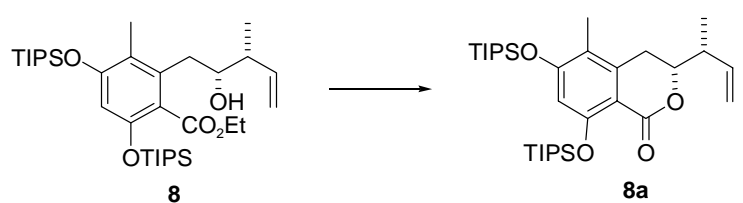

To a solution of compound $\mathbf{8}(6 \mathrm{~g}, 9.88 \mathrm{mmol})$ in DCM was added amberlyst resin $(1 \mathrm{~g})$. The reaction mixture was allowed to stir at room temperature for 2 hours. The resin was filtered off and the filtrate concentrated. The residue was purified by FC to give the desired product 8a $(5.54 \mathrm{~g}, 99 \%)$ as an oil. $[\alpha]^{20}{ }_{\mathrm{D}}=+35.65\left(\mathrm{c}=1.0\right.$, DCM). ${ }^{1} \mathrm{HNMR}$ $\left(\mathrm{CDCl}_{3}\right) \delta 6.30(\mathrm{~s}, 1 \mathrm{H}), 5.85-5.78(\mathrm{~m}, 1 \mathrm{H}), 5.14-5.10(\mathrm{~m}, 2 \mathrm{H}), 4.11-4.07(\mathrm{~m}, 1 \mathrm{H}), 2.85$ $(\mathrm{dd}, J=2.5,16.5 \mathrm{~Hz}, 1 \mathrm{H}), 2.62(\mathrm{dd}, J=11.0,16.0 \mathrm{~Hz}, 1 \mathrm{H}), 2.55(\mathrm{q}, J=6.5 \mathrm{~Hz}, 1 \mathrm{H}), 2.06$ (s, 3H), 1.35-1.26 (m, 6H), $1.19(\mathrm{~d}, J=6.5 \mathrm{~Hz}, 3 \mathrm{H}), 1.12-1.09(\mathrm{~m}, 36 \mathrm{H}) ;{ }^{13} \mathrm{C}$ NMR $\left(\mathrm{CDCl}_{3}\right) \delta$ 163.1, 159.0, 157.7, 141.0, 139.5, 118.3, 116.2, 110.1, 109.8, 79.7, 42.3, 29.6, 18.2, 16.0, 13.5, 13.3, 11.9; MS (ES) calculated for $\mathrm{C}_{32} \mathrm{H}_{57} \mathrm{O}_{4} \mathrm{Si}_{2}[\mathrm{M}+\mathrm{H}]^{+}: 561.38$, found 561.30 .

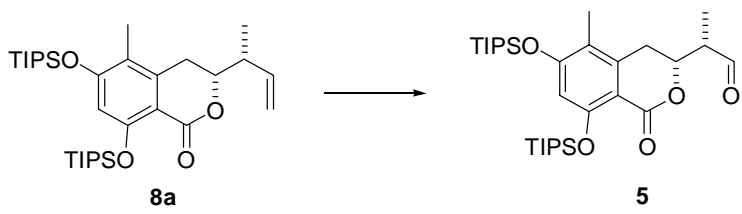

To a solution of $8 \mathbf{a}(969 \mathrm{mg}, 1.73 \mathrm{mmol})$ in THF/ $\mathrm{H}_{2} \mathrm{O}(10 / 1 \mathrm{ml})$ were added NMO (405 $\mathrm{mg}, 3.46 \mathrm{mmol})$ and $\mathrm{OsO}_{4}\left(2 \mathrm{ml}, 2.5 \% \mathrm{wt}\right.$ in $\left.{ }^{t} \mathrm{BuOH}, 0.173 \mathrm{mmol}\right)$ at $0{ }^{\circ} \mathrm{C}$. After stirring at ambient temperature for 12 hours, $10 \% \mathrm{Na}_{2} \mathrm{~S}_{2} \mathrm{O}_{3}(25 \mathrm{ml})$ solution was added. After 5 hours, the crude was extracted with EtOAc and the combined organic extracts were washed with water and dried over $\mathrm{MgSO}_{4}$. After concentration, the residue obtained was filtered through a short silica gel plug and washed with EtOAc. Removal of the solvent gave the crude diol. $\mathrm{NaIO}_{4}(1.11 \mathrm{~g}, 5.19 \mathrm{mmol})$ was added to a solution of crude diol in $90 \% \mathrm{MeOH} / \mathrm{H}_{2} \mathrm{O}(16 \mathrm{ml})$. After stirring at room temperature for 1 hour, EtOAc was added and the reaction mixture was washed with water and dried over $\mathrm{MgSO}_{4}$. After concentration, the residue was purified by FC (silica gel) to give the aldehyde 5 (876.6 $\mathrm{mg}, 90 \%)$ as a syrup. $[\alpha]^{20}{ }_{\mathrm{D}}=+58.40(\mathrm{c}=1.0, \mathrm{DCM}) .{ }^{1} \mathrm{HNMR}\left(\mathrm{CDCl}_{3}\right) \delta 9.83(\mathrm{~s}, 1 \mathrm{H})$, $6.31(\mathrm{~s}, 1 \mathrm{H}), 4.66-4.61(\mathrm{~m}, 1 \mathrm{H}), 4.12-4.08(\mathrm{~m}, 1 \mathrm{H}), 2.91(\mathrm{dd}, J=3.0,16.0 \mathrm{~Hz}, 1 \mathrm{H}), 2.79-$ 
$2.69(\mathrm{~m}, 2 \mathrm{H}), 2.07(\mathrm{~s}, 3 \mathrm{H}), 1.33-1.27(\mathrm{~m}, 9 \mathrm{H}), 1.13-1.10(\mathrm{~m}, 36 \mathrm{H}) ;{ }^{13} \mathrm{C} \mathrm{NMR}\left(\mathrm{CDCl}_{3}\right) \delta$ 202.7, 162.4, 159.3, 158.1, 140.3, 118.3, 109.9, 109.5, 75.7, 60.6, 50.0, 29.8, 21.2, 18.2, 18.1, 14.4, 13.5, 13.4, 12.0, 9.4; MS (ES) calculated for $\mathrm{C}_{31} \mathrm{H}_{55} \mathrm{O}_{5} \mathrm{Si}_{2}[\mathrm{M}+\mathrm{H}]^{+}$: 563.36, found 563.44 .

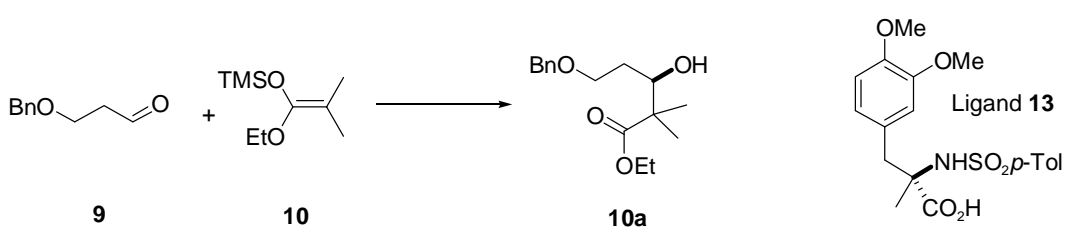

To a solution of ligand $\mathbf{1 3}(2.12 \mathrm{~g}, 5.4 \mathrm{mmol})$ in propylnitrile ( $40 \mathrm{ml})$ was added $\mathrm{BH}_{3} \mathrm{THF}$ complex $(5.4 \mathrm{ml}, 5.4 \mathrm{mmol})$ at $\mathrm{rt}$ and the mixture was stirred at $45{ }^{\circ} \mathrm{C}$ for 1 hour before cooled to $-78{ }^{\circ} \mathrm{C}$. Ketene acetal $10(7.71 \mathrm{~g}, 41 \mathrm{mmol})$ was then added at $-78{ }^{\circ} \mathrm{C}$, followed by the addition of aldehyde $9(4.43 \mathrm{~g}, 27 \mathrm{mmol})$ as a solution in propylnitrile $(10 \mathrm{ml})$ over 4 hours via syringe pump. The reaction mixture was stirred for another 2 hours before it was quenched with $\mathrm{pH} 7$ buffer $(50 \mathrm{ml})$. The reaction mixture was warmed to room temperature and diluted with EtOAc, the organic solution was washed with $\mathrm{NaHCO}_{3}$, dried over $\mathrm{Na}_{2} \mathrm{SO}_{4}$ and concentrated. The residue (TLC showed as a 1:1 mixture of TMS protected hydroxyl ester and free hydroxyl ester) was treated with $2.0 \mathrm{M} \mathrm{HCl} / \mathrm{Et}_{2} \mathrm{O}$ (2.0 eq.) at $0{ }^{\circ} \mathrm{C}$ for 1 hour at room temperature before it was directly concentrated and purified by FC to give 10a $(7.16 \mathrm{~g}, 95 \%)$. $[\alpha]^{20}{ }_{\mathrm{D}}=+15.95\left(\mathrm{c}=0.5\right.$, DCM). ${ }^{1} \mathrm{HNMR}$ $\left(\mathrm{CDCl}_{3}\right) \delta$ 7.34-7.26 (m, 5H), $4.53(\mathrm{~d}, J=12.0 \mathrm{~Hz}, 1 \mathrm{H}), 4.14(\mathrm{q}, J=7.0 \mathrm{~Hz}, 2 \mathrm{H}), 3.91$ $3.89(\mathrm{~m}, 1 \mathrm{H}), 3.75-3.65(\mathrm{~m}, 2 \mathrm{H}), 3.16(\mathrm{br}, 1 \mathrm{H}), 1.75-1.65(\mathrm{~m}, 2 \mathrm{H}), 1.26(\mathrm{t}, J=7.0 \mathrm{~Hz}$, $3 \mathrm{H}), 1.19(\mathrm{~s}, 3 \mathrm{H}), 1.17(\mathrm{~s}, 3 \mathrm{H}) ;{ }^{13} \mathrm{C} \mathrm{NMR}\left(\mathrm{CDCl}_{3}\right) \delta 177.5,138.2,128.6,128.4,128.0$, $127.8,77.7,75.5,73.5,69.4,60.7,47.0,31.7,21.8,20.6,18.1,14.3$.

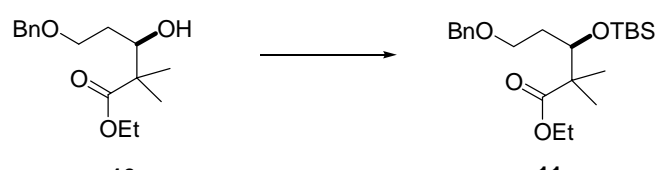

To a solution of compound 10a $(5.45 \mathrm{~g}, 19.4 \mathrm{mmol})$ in DCM (30 ml) was added 2, 6lutidine $(4.66 \mathrm{ml}, 40 \mathrm{mmol})$ at $0{ }^{\circ} \mathrm{C}$. After stirring for 5 minutes at $0{ }^{\circ} \mathrm{C}$, TBSOTf $(9.18$ $\mathrm{ml}, 40 \mathrm{mmol}$ ) was added dropwise. The reaction mixture was allowed to stir at room temperature for 2 hours. After the reaction was complete as judged by TLC, it was diluted with EtOAc. The organic layer was washed with $\mathrm{NaHCO}_{3}$, dried over $\mathrm{Na}_{2} \mathrm{SO}_{4}$ and concentrated. The residue was purified by FC (EtOAc/hexane 1/10) to give the TBS 
protected product $11(7.56 \mathrm{~g}, 99 \%)$ as an oil. $[\alpha]^{20}{ }_{\mathrm{D}}=+41.85(\mathrm{c}=1.0, \mathrm{DCM}) .{ }^{1} \mathrm{HNMR}$ $\left(\mathrm{CDCl}_{3}\right) \delta$ 7.36-7.26 (m, 5H), $4.48(\mathrm{dd}, J=12.0,15.5 \mathrm{~Hz}, 2 \mathrm{H}), 4.14-4.03(\mathrm{~m}, 3 \mathrm{H}), 3.55-$ $3.46(\mathrm{~m}, 2 \mathrm{H}), 1.80(\mathrm{ddt}, J=3.0,7.5,14.5 \mathrm{~Hz}), 1.66$ (ddt, $J=5.0,15.0,15.5 \mathrm{~Hz}, 1 \mathrm{H}$ ), 1.22 (t, $J=7.5 \mathrm{~Hz}, 3 \mathrm{H}), 1.15$ (s, 3H), 1.09 (s, 3H), 0.86 (s, 3H), 0.03 (s, 3H), 0.01 (s, $3 \mathrm{H}), ;{ }^{13} \mathrm{C} \mathrm{NMR}\left(\mathrm{CDCl}_{3}\right) \delta 177.2,138.7,128.5,127.8,127.7,74.0,73.0,67.9,60.6,48.4$, 34.2, 26.2, 22.1, 20.4, 18.5, 14.3, -3.8, -4.0; MS (ES) calculated for $\mathrm{C}_{22} \mathrm{H}_{39} \mathrm{O}_{4} \mathrm{Si}[\mathrm{M}+$ $\mathrm{H}]^{+}: 395.26$, found $395.24 ; \mathrm{C}_{22} \mathrm{H}_{38} \mathrm{NaO}_{4} \mathrm{Si}[\mathrm{M}+\mathrm{Na}]^{+}:$417.24, found 417.34.

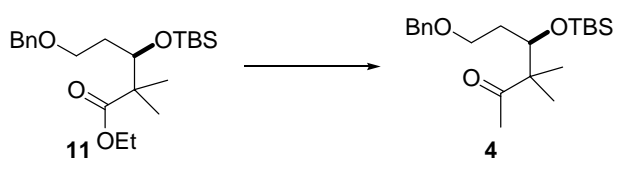

To a solution of compound $11(7.56 \mathrm{~g}, 19.2 \mathrm{mmol})$ in anhydrous pentane $(50 \mathrm{ml})$ was added trimethylsilyl methyl lithium $(58 \mathrm{ml}, 58.2 \mathrm{mmol})$ dropwise at $0{ }^{\circ} \mathrm{C}$. The mixture was stirred at $0{ }^{\circ} \mathrm{C}$ for 4 hours with TLC monitoring ( $\left.\mathrm{Et}_{2} \mathrm{O} / \mathrm{Hexane} 1 / 10\right)$. After the reaction was complete as judged by TLC, to this suspension was added dry methanol (10 $\mathrm{ml}$ ) and the resulting emulsion was stirred for another 1 hour at room temperature. The mixture was diluted with $\mathrm{Et}_{2} \mathrm{O}$ /water. Aqueous layer was extracted with $\mathrm{Et}_{2} \mathrm{O}$, dried over $\mathrm{Na}_{2} \mathrm{SO}_{4}$ and concentrated. The residue was purified by FC (EtOAc/Hexane 1/10) to give the methyl ketone $4(5.81 \mathrm{~g}, 82 \%)$ as an oil. $[\alpha]^{20}{ }_{\mathrm{D}}=+7.45\left(\mathrm{c}=1.0\right.$, DCM) ${ }^{1} \mathrm{HNMR}$ $\left(\mathrm{CDCl}_{3}\right) \delta$ 7.35-7.27 (m, 5H), $4.49(\mathrm{~d}, J=12.0 \mathrm{~Hz}, 1 \mathrm{H}), 4.45(\mathrm{~d}, J=12.0 \mathrm{~Hz}, 1 \mathrm{H}), 4.04$ (dd, $J=2.5,7.5 \mathrm{~Hz}, 1 \mathrm{H}), 3.50-3.48(\mathrm{~m}, 2 \mathrm{H}), 2.13(\mathrm{~s}, 3 \mathrm{H}), 1.78-1.72(\mathrm{~m}, 1 \mathrm{H}), 1.62-1.55$ (m, 1H), 1.10 (s, 3H), 1.06 (s, 3H), 0.87 (s, 9H), 0.05 (s, 3H), 0.03 (s, 3H); ${ }^{13} \mathrm{C}$ NMR $\left(\mathrm{CDCl}_{3}\right) \delta 213.7,138.7,128.6,127.7,74.1,73.1,67.7,53.5,34.5,27.1,26.2,22.1,20.3$, 18.5, -3.8; MS (ES) calculated for $\mathrm{C}_{21} \mathrm{H}_{37} \mathrm{O}_{3} \mathrm{Si}[\mathrm{M}+\mathrm{H}]^{+}$: 365.25, found 365.36; $\mathrm{C}_{21} \mathrm{H}_{36} \mathrm{NaO}_{3} \mathrm{Si}[\mathrm{M}+\mathrm{Na}]^{+}: 387.23$, found 387.09.

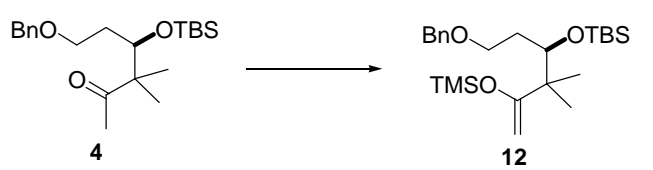

To a solution of compound $4(1.34 \mathrm{~g}, 3.6 \mathrm{mmol})$ in $\mathrm{DCM}(15 \mathrm{ml})$ at $0{ }^{\circ} \mathrm{C}$ was added $\mathrm{Et}_{3} \mathrm{~N}$ $(2 \mathrm{ml}, 14.4 \mathrm{mmol})$ and then TMSOTf $(1.3 \mathrm{ml}, 7.2 \mathrm{mmol})$. The reaction was allowed to stir at room temperature for 1 hour. The reaction mixture was diluted with hexane, washed with aq. $\mathrm{NaHCO}_{3}$ and dried over $\mathrm{Na}_{2} \mathrm{SO}_{4}$. The solution was directly passed through a short pad of silica gel plug eluted with EtOAc/Hexane (1/20). The combined 
organic solution was concentrated to give compound $12(1.58 \mathrm{~g}, 100 \%)$ as a light yellow oil. The crude 12 was used for the next step without any further purification. ${ }^{1} \mathrm{HNMR}$ $\left(\mathrm{CDCl}_{3}\right) \delta 7.33-7.25(\mathrm{~m}, 5 \mathrm{H}), 4.48(\mathrm{~s}, 2 \mathrm{H}), 4.11(\mathrm{~s}, 1 \mathrm{H}), 3.95(\mathrm{~s}, 1 \mathrm{H}), 3.87(\mathrm{dd}, J=3.0$, 8.0 Hz, 1H), 3.55-3.45 (m, 2H), 1.93-1.86 (m, 1H), 1.61-1.54 (m, 1H), 1.02 (s, 3H), 0.94 $(\mathrm{s}, 3 \mathrm{H}), 0.87(\mathrm{~s}, 9 \mathrm{H}), 0.18(\mathrm{~s}, 9 \mathrm{H}), 0.03(\mathrm{~s}, 3 \mathrm{H}), 0.01(\mathrm{~s}, 3 \mathrm{H}) ;{ }^{13} \mathrm{C} \mathrm{NMR}\left(\mathrm{CDCl}_{3}\right) \delta 165.0$, 139.0, 128.5, 127.8, 127.6, 88.2, 73.2, 72.9, 68.6, 45.4, 33.8, 26.4, 24.7, 19.8, 18.6, 0.28, -3.8 .

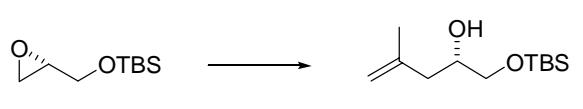

14

$14 a$

To a solution of compound $14(8.87 \mathrm{~g}, 47.2 \mathrm{mmol})$ in anhydrous THF (150 ml) was added $\mathrm{CuI}(2.3 \mathrm{~g}, 11.8 \mathrm{mmol})$ and the resulting suspension was cooled to $-15{ }^{\circ} \mathrm{C}$. Vinylmagnesium bromide (180 ml, $0.5 \mathrm{M}$ in THF, $94.4 \mathrm{mmol})$ was added dropwise through an addition funnel under $\mathrm{N}_{2}$. The reaction mixture was allowed to stir at $-15^{\circ} \mathrm{C}$ for 3 hours with TLC monitoring before it was quenched by aq. $\mathrm{NH}_{4} \mathrm{Cl}(100 \mathrm{ml})$ and diluted with $\mathrm{Et}_{2} \mathrm{O}(200 \mathrm{ml})$. The organic layer was washed with brine, dried $\left(\mathrm{Na}_{2} \mathrm{SO}_{4}\right)$ and concentrated. The residue was purified by FC (Hexane to EtOAc/Hexane 1/30) to give the alcohol 14a $(8.9 \mathrm{~g}, 82 \%)$ as an oil. ${ }^{1} \mathrm{HNMR}\left(\mathrm{CDCl}_{3}\right) \delta 4.83(\mathrm{~s}, 1 \mathrm{H}), 4.78(\mathrm{~s}, 1 \mathrm{H}), 3.83-$ $3.78(\mathrm{~m}, 1 \mathrm{H}), 3.61(\mathrm{dd}, J=3.5,10.0 \mathrm{~Hz}, 1 \mathrm{H}), 3.46(\mathrm{dd}, J=7.0,10.0 \mathrm{~Hz}, 1 \mathrm{H}), 2.37$ (d, $J=$ $3.5 \mathrm{~Hz}, 1 \mathrm{H}), 2.17(\mathrm{~d}, J=6.5 \mathrm{~Hz}, 2 \mathrm{H}), 1.77$ (s, 3H), 0.90 (s, 3H), 0.07 (s, 3H); ${ }^{13} \mathrm{C} \mathrm{NMR}$ $\left(\mathrm{CDCl}_{3}\right) \delta 142.6,113.1,69.8,67.0,41.8,26.1,22.8,18.5,-5.2$.

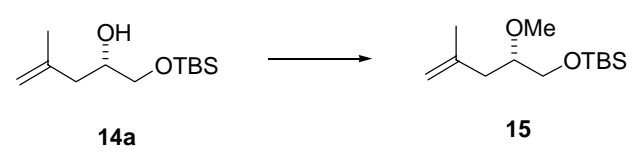

To a solution of compound 14a (30 g, $130 \mathrm{mmol})$ in anhydrous DCM (300 ml) was added freshly activated 4A MS (5 g, powder), proton sponge (50 g, $234 \mathrm{mmol})$ and $\mathrm{Me}_{3} \mathrm{OBF}_{4}$ $(23 \mathrm{~g}, 156 \mathrm{mmol})$. The suspension was allowed to stir at room temperature overnight before it was diluted with DCM and filtered. The filtrate was washed with water, aq. $\mathrm{HCl}$, $\mathrm{NaHCO}_{3}$, and brine. The organic layer was concentrated and the residue was purified by FC (Hexane to $\mathrm{Et}_{2} \mathrm{O} /$ Hexane $\left.1 / 20\right)$ to give $15(30 \mathrm{~g}, 95 \%)$ as a volatile oil: ${ }^{1} \mathrm{HNMR}$ $\left(\mathrm{CDCl}_{3}\right) \delta 4.80(\mathrm{~s}, 1 \mathrm{H}), 4.75(\mathrm{~s}, 1 \mathrm{H}), 3.62(\mathrm{dd}, J=6.0,10.5 \mathrm{~Hz}, 1 \mathrm{H}), 3.58(\mathrm{dd}, J=4.5$, $10.5 \mathrm{~Hz}, 1 \mathrm{H}), 3.42$ (s, 3H), 3.41-3.36 (m, 1H), 2.23 (dd, $J=5.5,14.5 \mathrm{~Hz}, 1 \mathrm{H}), 2.16$ (dd, $J$ 
= 7.5, $14.5 \mathrm{~Hz}, 1 \mathrm{H}), 1.77(\mathrm{~s}, 3 \mathrm{H}), 0.90(\mathrm{~s}, 9 \mathrm{H}), 0.06(\mathrm{~s}, 6 \mathrm{H}) ;{ }^{13} \mathrm{C} \mathrm{NMR}\left(\mathrm{CDCl}_{3}\right) \delta 143.0$, 112.6, 80.6, 65.1, 58.1, 40.0, 26.1, 23.1, 18.5, -5.1, -5.2. MS (ES) calculated for $\mathrm{C}_{13} \mathrm{H}_{29} \mathrm{O}_{2} \mathrm{Si}[\mathrm{M}+\mathrm{H}]^{+}: 245.19$, found 245.18 .

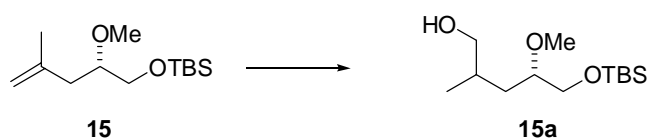

A solution of compound $15(5 \mathrm{~g}, 20.4 \mathrm{mmol})$ in THF $(50 \mathrm{ml})$ was treated with $\mathrm{BH}_{3} \mathrm{THF}$ (22.5 ml, 1.0 M in THF, $22.5 \mathrm{mmol}$ ) solution at $0{ }^{\circ} \mathrm{C}$ for 1 hour. To this solution was added aq. $\mathrm{NaOH}(1 \mathrm{~N}, 100 \mathrm{ml})$ solution followed immediately by aq. $30 \% \mathrm{H}_{2} \mathrm{O}_{2}(11 \mathrm{ml})$. The ice bath was then removed and the mixture was allowed to stir at room temperature for another hour before diluted with EtOAc. The solution was washed with water and brine, dried $\left(\mathrm{MgSO}_{4}\right)$ and concentrated. FC (EtOAc/Hexane 1/4) of the residue gave compound 15a $(4.7 \mathrm{~g}, 88 \%)$ as a pair of diastereomers. ${ }^{1} \mathrm{HNMR}\left(\mathrm{CDCl}_{3}\right) \delta 3.68-3.26(\mathrm{~m}$, $5 \mathrm{H}$, both isomers), 3.43 (s, 3H, isomer I), 3.42 (s, 3H, isomer II), 2.83 (br, 1H, isomer II), $2.66(\mathrm{br}, 1 \mathrm{H}$, isomer $\mathrm{I}), 1.89-1.76(\mathrm{~m}, 1 \mathrm{H}$, both isomers $), 1.56-1.36(\mathrm{~m}, 2 \mathrm{H}$, both isomers), $0.93(\mathrm{~d}, J=7.0 \mathrm{~Hz}, 3 \mathrm{H}$, isomer $\mathrm{I}), 0.90$ (d, $J=7.0 \mathrm{~Hz}, 3 \mathrm{H}$, isomer II), 0.89 (s, 9H, isomer I), 0.88 (s, 9H, isomer II), $0.05(\mathrm{~s}, 6 \mathrm{H}) ;{ }^{13} \mathrm{C} \mathrm{NMR}\left(\mathrm{CDCl}_{3}\right) \delta 81.1,80.0,68.6$, $68.1,65.5,65.0,58.1,57.8,36.6,35.7,33.7,32.5,26.0,18.4,18.0,17.7,-5.2,-5.3$.

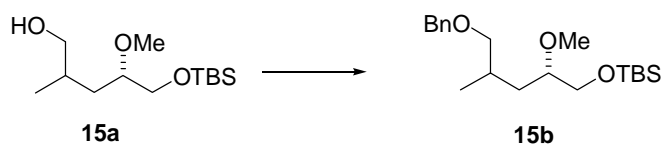

To a solution of compound 15a $(1.9 \mathrm{~g}, 5.2 \mathrm{mmol})$ in anhydrous DMF $(15 \mathrm{ml})$ was added $\mathrm{NaH}$ (380mg, $10 \mathrm{mmol})$ at $0{ }^{\circ} \mathrm{C}$, after stirring for 15 minutes, $\mathrm{BnBr}(1.18 \mathrm{ml}, 10 \mathrm{mmol})$ was added .The reaction was allowed to stir at $\mathrm{rt}$ for 2 hours. The reaction mixture was quenched by aq. $\mathrm{NH}_{4} \mathrm{Cl}$, extracted with EtOAc, washed with aq. $\mathrm{NaHCO}_{3}$ and brine. The organic layer was dried $\left(\mathrm{Na}_{2} \mathrm{SO}_{4}\right)$ and concentrated. The residue was purified by $\mathrm{FC}$ (Hexane to $\left.\mathrm{Et}_{2} \mathrm{O} / \mathrm{Hexane} 1 / 20\right)$ to give compound $\mathbf{1 5 b}(1.68 \mathrm{~g}, 92 \%)$ as an oil. ${ }^{1} \mathrm{HNMR}$ $\left(\mathrm{CDCl}_{3}\right) \delta 7.40-7.38(\mathrm{~m}, 5 \mathrm{H}), 4.57(\mathrm{~s}, 2 \mathrm{H}), 3.72-3.30(\mathrm{~m}, 5 \mathrm{H}), 3.48(\mathrm{~s}, 3 \mathrm{H}$, isomer $\mathrm{I}), 3.46$ (s, 3H, isomer II), 2.10-1.99 (m, 1H), 1.65-1.56 (m, 1H), 1.39-1.30 (m, 1H), $1.06(\mathrm{~d}, J=$ 7.0, 3H, isomer I), $1.04\left(\mathrm{~d}, J=7.0 \mathrm{~Hz}, 3 \mathrm{H}\right.$, isomer II), $0.96(\mathrm{~s}, 9 \mathrm{H}), 0.12(\mathrm{~s}, 6 \mathrm{H}) ;{ }^{13} \mathrm{C}$ NMR $\left(\mathrm{CDCl}_{3}\right) \delta 139.0,128.6,127.8,127.7,127.6,80.6,80.0,76.5,75.9,73.2,73.1$, $66.0,65.8,58.4,58.1,36.2,36.1,30.7,30.3,26.2,18.6,18.5,17.4,-5.1$. 


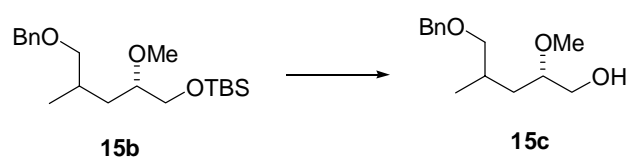

To a solution of compound $\mathbf{1 5 b}(1.68 \mathrm{~g}, 4.77 \mathrm{mmol})$ in THF $(10 \mathrm{ml})$ was added TBAF (10 ml, $1.0 \mathrm{M}$ in THF) at room temperature. The reaction mixture was allowed to stir at $\mathrm{rt}$ overnight before it was diluted with EtOAc. The mixture was washed with water and brine. The organic layer was dried $\left(\mathrm{Na}_{2} \mathrm{SO}_{4}\right)$ and concentrated. The residue was purified by FC (Hexane to EtOAc/Hexane 1/5) to give compound 15c $(1.14 \mathrm{~g}, 99 \%)$ as an oil. ${ }^{1} \mathrm{HNMR}\left(\mathrm{CDCl}_{3}\right) \delta$ 7.36-7.26 (m, 5H), $4.50(\mathrm{~s}, 2 \mathrm{H}), 3.71-3.27(\mathrm{~m}, 8 \mathrm{H}), 2.13-1.19(\mathrm{~m}$, $3 \mathrm{H}), 0.98(\mathrm{~d}, J=7.0 \mathrm{~Hz}, 3 \mathrm{H}) ;{ }^{13} \mathrm{C} \mathrm{NMR}\left(\mathrm{CDCl}_{3}\right) \delta 138.9,138.8,128.6,127.8,80.2,79.7$, 76.2, 76.0, 73.3, 64.3, 64.1, 57.4, 57.1, 35.3, 34.8, 30.6, 30.4, 18.0, 17.9.

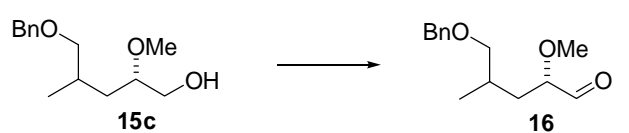

A solution of oxylyl chloride $(3 \mathrm{ml}, 35 \mathrm{mmol})$ in DCM $(50 \mathrm{ml})$ at $-78{ }^{\circ} \mathrm{C}$ was added DMSO (4.97 ml, $70 \mathrm{mmol})$ slowly. 5 minutes later, alcohol $15 \mathrm{c}(4.25 \mathrm{~g}, 17.8 \mathrm{mmol})$ in DCM $(30 \mathrm{ml})$ was added. $\mathrm{Et}_{3} \mathrm{~N}(9.7 \mathrm{ml}, 70 \mathrm{mmol})$ was added at $-78{ }^{\circ} \mathrm{C}$ in 45 minutes. The reaction mixture was allowed to stir at $-78{ }^{\circ} \mathrm{C}$ for 15 minutes before the cold bath was removed. The reaction was kept stirring from $-78{ }^{\circ} \mathrm{C}$ to $\mathrm{rt}$, then at $\mathrm{rt}$ for $0.5 \mathrm{~h}$. The reaction mixture was diluted with EtOAc, washed with $\mathrm{NaHCO}_{3}$, brine, dried and concentrated. The residue was purified by FC (EtOAc/Hexane 1/9) to give aldehyde 16 (3.95 g, 94\%) as a yellow oil. ${ }^{1} \mathrm{HNMR}\left(\mathrm{CDCl}_{3}\right) \delta 9.64(\mathrm{~d}, J=2.0 \mathrm{~Hz}$, isomer I), $9.61(\mathrm{~d}, J$ $=2.0 \mathrm{~Hz}, 1 \mathrm{H}$, isomer II $), 7.36-7.26(\mathrm{~m}, 5 \mathrm{H}), 4.52-4.47(\mathrm{~m}, 2 \mathrm{H}), 3.68-3.64(\mathrm{~m}, 1 \mathrm{H}), 3.43$ (s, 3H, isomer I), 3.39 (s, 3H, isomer II), 3.34-3.31 (m, 2H), 2.07-1.98 (m, 1H), 1.83-1.75 $(\mathrm{m}, 1 \mathrm{H}), 1.51-1.41(\mathrm{~m}, 1 \mathrm{H}), 0.98(\mathrm{~d}, J=7.5 \mathrm{~Hz}, 3 \mathrm{H}) ;{ }^{13} \mathrm{C} \mathrm{NMR}\left(\mathrm{CDCl}_{3}\right) \delta 204.0,203.8$, 138.7, 138.6, 128.5, 127.7, 127.6, 84.6, 84.1, 75.6, 75.2, 73.2, 73.0, 58.5, 58.3, 34.0, 33.7, 30.0, 29.8, 18.1, 16.9; MS (ES) calculated for $\mathrm{C}_{14} \mathrm{H}_{21} \mathrm{O}_{3}[\mathrm{M}+\mathrm{H}]^{+}: 237.15$, found 237.12.
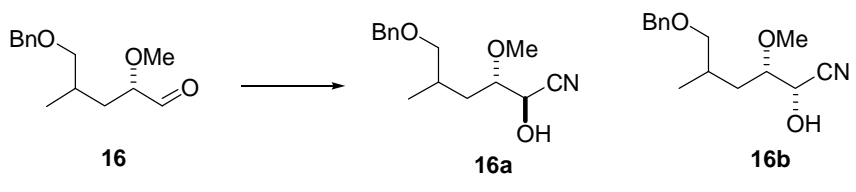

To a solution of aldehyde $16(4 \mathrm{~g}, 17 \mathrm{mmol})$ in DCM $(100 \mathrm{ml})$ was added TMSCN $(5.6$ $\mathrm{ml}, 42.5 \mathrm{mmol}$ ) at $0{ }^{\circ} \mathrm{C}, \mathrm{AlCl}_{3}$ was added 5 minutes later. The reaction mixture was stirred from $0{ }^{\circ} \mathrm{C}$ to room temperature overnight. The reaction was quenched by adding 
aq $\mathrm{NaHCO}_{3}$ solution and extracted with EtOAc. The organic layer was washed with brine, dried and concentrated. The residue was purified by FC (silica gel, Acetone/Hexane 1/4) to give $\mathbf{1 6 a}$ and $\mathbf{1 6 b}(3.88 \mathrm{~g}, 87 \%)$ as an inseparable mixture (16a/16b, 2/1). ${ }^{1} \mathrm{HNMR}\left(\mathrm{CDCl}_{3}\right) \delta$ 7.38-7.28 (m, 5H), 4.55-4.34 (m, 3H), 3.65-3.53 (m, 1H), 3.39-3.26 (m, 2H), 3.53, 3.51 (s, 3H, 16b), 3.46-3.45 (s, 3H, 16a) 2.01-1.43 (m, 3H), 1.00-0.97 (m, 3H); MS (ES) calculated for $\mathrm{C}_{15} \mathrm{H}_{22} \mathrm{NO}_{3}[\mathrm{M}+\mathrm{H}]^{+}:$264.16, found 264.10.

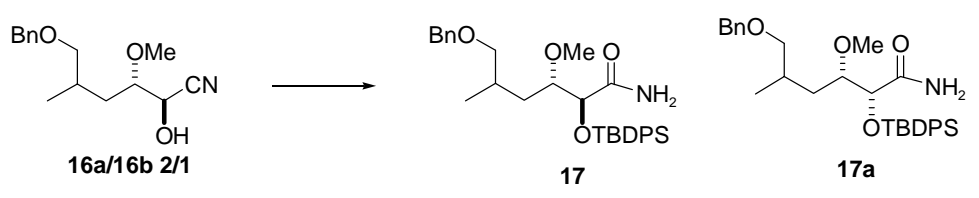

To a solution of compound 16a/16b $(1.23 \mathrm{~g}, 4.68 \mathrm{mmol})$ in DCM (15 ml) was added $\mathrm{NEt}_{3}$ $(1.15 \mathrm{ml}, 8.24 \mathrm{mmol})$, DMAP $(61 \mathrm{mg}, 0.5 \mathrm{mmol})$ at $0{ }^{\circ} \mathrm{C}$. To the solution was added TBDPSCl $(1.82 \mathrm{ml}, 7 \mathrm{mmol})$. The reaction mixture was stirred at $\mathrm{rt}$ overnight before being diluted with EtOAc and washed with aq. $\mathrm{NH}_{4} \mathrm{Cl}, \mathrm{NaHCO}_{3}$ and brine sequentially. After dried over $\mathrm{Na}_{2} \mathrm{SO}_{4}$, it was concentrated and purified by $\mathrm{FC}\left(\mathrm{Et}_{2} \mathrm{O} / \mathrm{Hexane} 1 / 10\right)$ to give the protected cyanohydrin. To a solution of this cyanohydrin in $\mathrm{H}_{2} \mathrm{O} / \mathrm{THF}(16 \mathrm{ml}$, 1/3) was added $\mathrm{PdCl}_{2}(177 \mathrm{mg})$ followed by acetamide $(1.18 \mathrm{~g}, 20 \mathrm{mmol})$. The reaction mixture was stirred vigorously at $\mathrm{rt}$ for 10 hours before it was diluted with EtOAc and washed with $\mathrm{NaHCO}_{3}$ and brine. After being dried over $\mathrm{Na}_{2} \mathrm{SO}_{4}$, it was concentrated and purified by FC (EtOAc/Hexane 1/4) to give isolated pure compound 17 (1.3 g, 53\% over two steps) as the major product $(\mathbf{1 7 : 1 7 a}=2: 1) .{ }^{1} \mathrm{HNMR}\left(\mathrm{CDCl}_{3}\right) \delta 7.71-7.31(\mathrm{~m}, 15 \mathrm{H})$, $6.58(\mathrm{br}, 1 \mathrm{H}), 5.35(\mathrm{br}, 1 \mathrm{H}), 4.47(\mathrm{~s}, 1 \mathrm{H}), 4.44(\mathrm{~d}, J=2.5 \mathrm{~Hz}, 1 \mathrm{H}), 4.38,4.34$ (d, $J=2.0$ $\mathrm{Hz}, 1 \mathrm{H}), 3.38(\mathrm{~m}, 1 \mathrm{H}), 3.29,3.26(\mathrm{dd}, J=5.5,9.0 \mathrm{~Hz}, 1 \mathrm{H}), 3.19,3.13(\mathrm{dd}, J=6.5,9.0$ $\mathrm{Hz}, 1 \mathrm{H}), 3.11(\mathrm{~s}, 3 \mathrm{H}), 1.90,1.83(\mathrm{~m}, 1 \mathrm{H}), 1.59,1.45(\mathrm{~m}, 1 \mathrm{H}), 1.10,1.04(\mathrm{~m}, 1 \mathrm{H}), 1.13$ (s, $9 \mathrm{H}), 0.88,0.82(\mathrm{~d}, J=6.5 \mathrm{~Hz}, 3 \mathrm{H})$; MS (ES) calculated for $\mathrm{C}_{31} \mathrm{H}_{42} \mathrm{NO}_{4} \mathrm{Si}[\mathrm{M}+\mathrm{H}]^{+}$: 520.29 , found 520.35 .

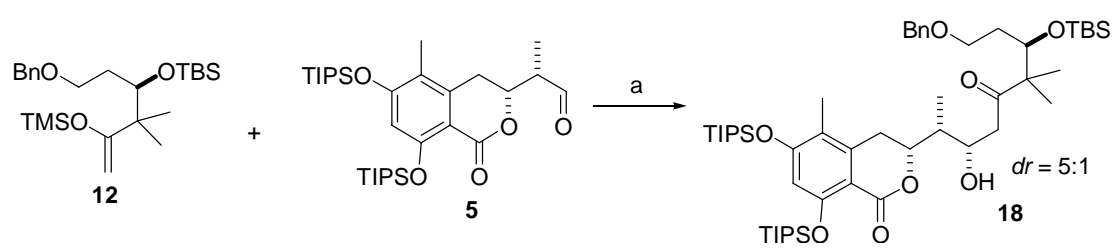


A solution of aldehyde $\mathbf{5}(910 \mathrm{mg}, 1.7 \mathrm{mmol})$ and silylenolether $\mathbf{1 2}(1.58 \mathrm{~g}, 3.6 \mathrm{mmol})$ was azeotropically dried with toluene several times before dissolved in anhydrous DCM (25 $\mathrm{ml})$. The solution was cooled to $-78{ }^{\circ} \mathrm{C}$ and freshly distilled $\mathrm{BF}_{3} \mathrm{OEt}_{2}(2.2 \mathrm{ml}, 18 \mathrm{mmol})$ was added dropwise. The reaction mixture was allowed to stir at $-78{ }^{\circ} \mathrm{C}$ overnight before it was quenched by addition of saturated $\mathrm{NaHCO}_{3}(30 \mathrm{ml})$ at $-78{ }^{\circ} \mathrm{C}$ and extracted with EtOAc. Combined organic layers were washed with brine and dried over $\mathrm{Na}_{2} \mathrm{SO}_{4}$. After concentration, the residue was purified by FC (EtOAc/Hexane 1/9) to give product 18 ( $0.96 \mathrm{~g}$ as pure isomer, $76 \%$, (for two isomers: $1.15 \mathrm{~g}, 91 \%, d r=5: 1)$ ) as a colorless oil. The excess enolether was recovered as the hydroxyketone 4. $[\alpha]^{20}{ }_{D}=+28.59(\mathrm{c}=0.1$, DCM); ${ }^{1} \mathrm{HNMR}\left(\mathrm{CDCl}_{3}\right) \delta$ 7.34-7.26 (m, 5H), $6.30(\mathrm{~s}, 1 \mathrm{H}), 4.48(\mathrm{~d}, J=12.0 \mathrm{~Hz}, 1 \mathrm{H})$, $4.44(\mathrm{~d}, J=12.0 \mathrm{~Hz}, 1 \mathrm{H}), 4.36$ (ddd, $J=2.5,5.0,12.5 \mathrm{~Hz}, 1 \mathrm{H}), 4.31-4.27(\mathrm{~m}, 1 \mathrm{H}), 4.05$ (dd, $J=2.5,7.5 \mathrm{~Hz}, 1 \mathrm{H}), 3.49(\mathrm{dd}, J=5.5,8.0 \mathrm{~Hz}, 2 \mathrm{H}), 3.24(\mathrm{~d}, J=2.0 \mathrm{~Hz}, 1 \mathrm{H}), 2.89$ (dd, $J=2.5,16.0 \mathrm{~Hz}, 1 \mathrm{H}), 2.78-2.71(\mathrm{~m}, 2 \mathrm{H}), 2.08$ (s, 3H), 1.83-1.69 (m, 2H), 1.62-1.54 (m, 5H), 1.35-1.25 (m, 8H), 1.18-1.05 (m, $42 \mathrm{H}), 0.86$ (s, 9H), 0.05 (s, 3H), 0.04 (s, 3H); ${ }^{13} \mathrm{C} \mathrm{NMR}\left(\mathrm{CDCl}_{3}\right) \delta 217.4,163.1,159.0,157.7,141.4,138.5,128.6,127.9,118.3,109.7$, 78.3, 74.0, 73.2, 67.9, 67.5, 53.8, 42.9, 41.7, 34.7, 29.9, 26.2, 22.3, 20.2, 18.5, 18.3, 18.2, $17.9,13.5,13.4,12.0,9.6,-3.7,-3.8$; MS (ES) calculated for $\mathrm{C}_{52} \mathrm{H}_{91} \mathrm{O}_{8} \mathrm{Si}_{3}[\mathrm{M}+\mathrm{H}]^{+}$: 927.60, found 927.73; $\mathrm{C}_{52} \mathrm{H}_{90} \mathrm{NaO}_{8} \mathrm{Si}_{3}[\mathrm{M}+\mathrm{Na}]^{+}$: 949.58, found 949.68;

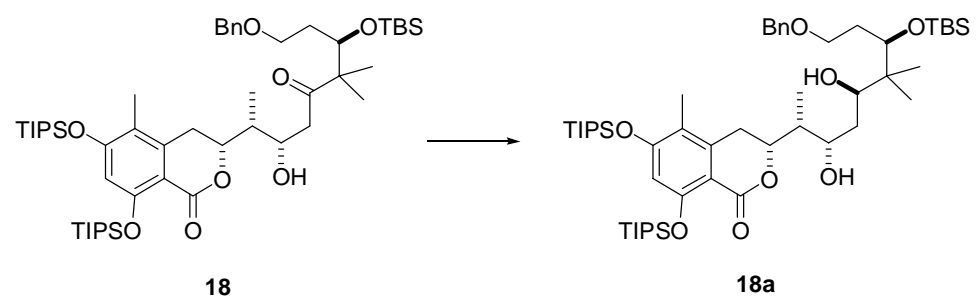

To a solution of compound 18 (1.15 g, $1.54 \mathrm{mmol})$ in anhydrous THF (30 ml) was added catecholborane (20 ml, $1.0 \mathrm{M}$ in THF, $20 \mathrm{mmol}$ ) at $-78{ }^{\circ} \mathrm{C}$. After stirring at $0{ }^{\circ} \mathrm{C}$ for $15 \mathrm{~h}$, the reaction was quenched by $10 \mathrm{ml}$ anhydrous $\mathrm{MeOH}$ and aq. solution of sodium potassium tartrate $(50 \mathrm{ml})$. The mixture was further stirred at $\mathrm{rt}$ for 1 hour before a normal workup process with EtOAc. The residue was purified by FC (EtOAc/Hexane 1/8) to give the desired product 18a $(1.05 \mathrm{~g}, 92 \%$ as a mixture of $15: 1$ isomers, $d r=$ 15:1). $[\alpha]^{20}{ }_{\mathrm{D}}=+44.37(\mathrm{c}=0.1, \mathrm{DCM}) ;{ }^{1} \mathrm{HNMR}\left(\mathrm{CDCl}_{3}\right) \delta$ 7.37-7.28 (m, 5H), $6.30(\mathrm{~s}$, 1H), 4.87 (br, 1H), 4.51 (d, $J=12.0 \mathrm{~Hz}, 1 \mathrm{H}), 4.48(\mathrm{~d}, J=12.0 \mathrm{~Hz}, 1 \mathrm{H}), 4.35$ (ddd, $J=$ 
2.5, 5.5, $12.5 \mathrm{~Hz}, 1 \mathrm{H}), 4.25(\mathrm{br}, 1 \mathrm{H}), 4.14(\mathrm{~d}, J=10.0 \mathrm{~Hz}, 1 \mathrm{H}), 4.06(\mathrm{~d}, J=9.5 \mathrm{~Hz}, 1 \mathrm{H})$, $3.66(\mathrm{dd}, J=2.5,7.5 \mathrm{~Hz}, 1 \mathrm{H}), 3.60-3.52(\mathrm{~m}, 2 \mathrm{H}), 2.98(\mathrm{dd}, J=3.0,16.5 \mathrm{~Hz}, 1 \mathrm{H}), 2.80$ $(\mathrm{dd}, J=12.0,16.0 \mathrm{~Hz}, 1 \mathrm{H}), 2.10(\mathrm{~s}, 3 \mathrm{H}), 2.11-2.09(\mathrm{~m}, 1 \mathrm{H}), 1.87-1.78(\mathrm{~m}, 2 \mathrm{H}), 1.66-1.59$ $(\mathrm{m}, 1 \mathrm{H}), 1.35-1.25(\mathrm{~m}, 6 \mathrm{H}), 1.17-1.11(\mathrm{~m}, 39 \mathrm{H}), 1.02(\mathrm{~s}, 3 \mathrm{H}), 0.87(\mathrm{~s}, 9 \mathrm{H}), 0.75(\mathrm{~s}, 3 \mathrm{H})$, $0.10(\mathrm{~s}, 3 \mathrm{H}), 0.07$ (s, $3 \mathrm{H}) ;{ }^{13} \mathrm{C}$ NMR $\left(\mathrm{CDCl}_{3}\right) \delta 163.5,158.9,157.6,141.8,138.6,128.6$, 127.9, 118.4, 109.7, 81.3, 78.7, 77.9, 73.1, 72.7, 67.6, 43.1, 40.8, 35.0, 33.3, 29.9, 26.2, 23.5, 21.0, 18.4, 18.3, 18.2, 13.5, 13.4, 12.1, 9.9, -3.8, -4.1; MS (ES) calculated for $\mathrm{C}_{52} \mathrm{H}_{93} \mathrm{O}_{8} \mathrm{Si}_{3}[\mathrm{M}+\mathrm{H}]^{+}:$929.62, found 929.68; $\mathrm{C}_{52} \mathrm{H}_{92} \mathrm{NaO}_{8} \mathrm{Si}_{3}[\mathrm{M}+\mathrm{Na}]^{+}: 951.60$, found 951.66.

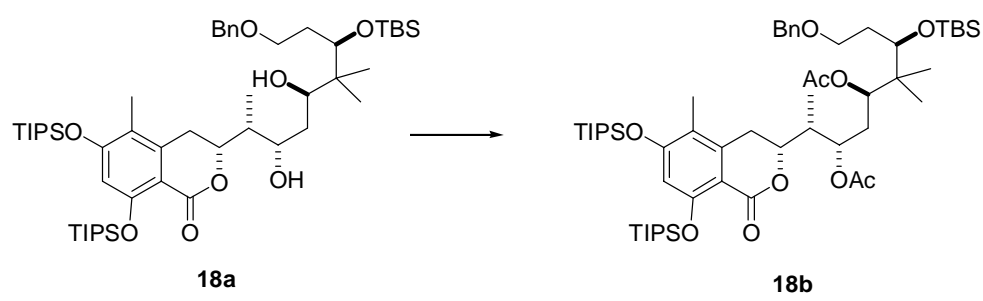

To a solution of compound 18a $(2.14 \mathrm{~g}, 2.3 \mathrm{mmol})$ in pyridine $(4 \mathrm{ml})$ was added $\mathrm{Ac}_{2} \mathrm{O}$ (2 $\mathrm{ml}$ ) and DMAP (35 mg, $0.287 \mathrm{mmol}$ ) at $0{ }^{\circ} \mathrm{C}$. The reaction mixture was allowed to stir at rt for $8 \mathrm{~h}$ before it was diluted with EtOAc. The mixture was washed with aq. $\mathrm{HCl}$, $\mathrm{NaHCO}_{3}$, brine sequentially. After being dried over $\mathrm{Na}_{2} \mathrm{SO}_{4}$, it was concentrated and purified by FC (EtOAc/Hexane 1/10 to 1/4) to give 18b (1.82 $\mathrm{g}, 78 \%$ ) as a pure isomer as an oil. ${ }^{1} \mathrm{HNMR}\left(\mathrm{CDCl}_{3}\right) \delta$ 7.33-7.25 (m, 5H), $6.28(\mathrm{~s}, 1 \mathrm{H}), 5.09(\mathrm{~m}, 1 \mathrm{H}), 4.98-4.95(\mathrm{~m}$, $1 \mathrm{H}), 4.50(\mathrm{~d}, J=12.0 \mathrm{~Hz}, 1 \mathrm{H}), 4.46(\mathrm{~d}, J=12.0 \mathrm{~Hz}, 1 \mathrm{H}), 4.02(\mathrm{ddd}, J=2.5,7.0,12.0 \mathrm{~Hz}$, $1 \mathrm{H}), 3.64(\mathrm{dd}, J=2.0,8.0 \mathrm{~Hz}, 1 \mathrm{H}), 3.53-3.50(\mathrm{~m}, 2 \mathrm{H}), 2.92(\mathrm{dd}, J=2.5,16.0 \mathrm{~Hz}, 1 \mathrm{H})$, $2.58(\mathrm{dd}, J=12.0,16.0 \mathrm{~Hz}, 1 \mathrm{H}), 2.09$ (s, 3H), 2.08 (s, 3H), 2.00 (s, 3H), 2.04-2.00 (m, $1 \mathrm{H}), 1.95-1.87(\mathrm{~m}, 1 \mathrm{H}), 1.73-1.58(\mathrm{~m}, 2 \mathrm{H}), 1.35-1.25(\mathrm{~m}, 6 \mathrm{H}), 1.16-1.10(\mathrm{~m}, 39 \mathrm{H}), 0.89$ (s, 3H), 0.88 (s, 3H), 0.83 (s, 3H), 0.04 (s, 3H), 0.03 (s, 3H); ${ }^{13} \mathrm{C}$ NMR $\left(\mathrm{CDCl}_{3}\right) \delta$ 171.1, 170.7, 162.9, 158.9, 157.6, 141.2, 138.6, 128.5, 128.0, 127.8, 118.3, 109.9, 109.7, 78.2, 74.7, 74.0, 73.2 , 71.7, 67.5, 42.9, 39.6, 33.7, 32.4, 30.5, 26.4, 21.4, 21.3, 20.7, 19.6, 18.7, 18.2, 13.5, 13.3, 11.8, 8.9, -3.7; MS (ES) calculated for $\mathrm{C}_{56} \mathrm{H}_{97} \mathrm{O}_{10} \mathrm{Si}_{3}[\mathrm{M}+\mathrm{H}]^{+}$: 1013.64, found 1013.70; $\mathrm{C}_{56} \mathrm{H}_{96} \mathrm{NaO}_{10} \mathrm{Si}_{3}[\mathrm{M}+\mathrm{Na}]^{+}$: 1035.62, found 1035.72. 


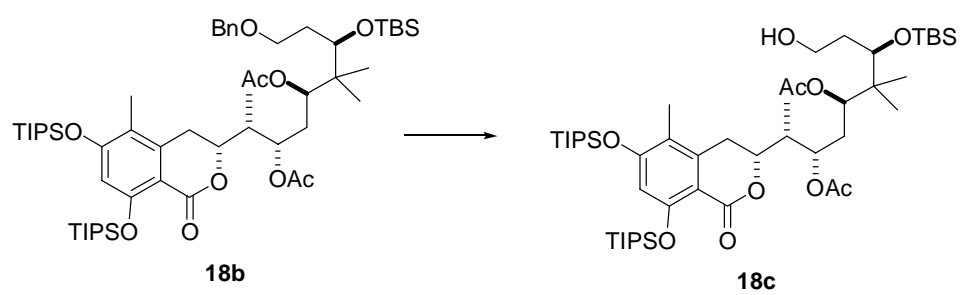

To a solution of compound $\mathbf{1 8 b}(1.82 \mathrm{~g}, 1.79 \mathrm{mmol})$ in EtOH $(15 \mathrm{ml})$ was charged $\mathrm{Pd} / \mathrm{C}$ $(180 \mathrm{mg})$ and the flask was sealed with rubber stopper. The inner atmosphere was exchanged three times with hydrogen before it was allowed to stir under $\mathrm{H}_{2}$ (double layer balloon) overnight. The reaction mixture was filtered through a celite pad followed by washing with EtOH twice. The combined organic solution was concentrated and purified by $\mathrm{FC}(\mathrm{E} / \mathrm{H} 1 / 4)$ to give the alcohol 18c $(1.65 \mathrm{~g}, 99 \%)$ as an oil: ${ }^{1} \mathrm{HNMR}\left(\mathrm{CDCl}_{3}\right) \delta 6.29$ (s, 1H), 5.10-5.07 (m, 1H), $4.98(\mathrm{dd}, J=2.0,10.0 \mathrm{~Hz}, 1 \mathrm{H}), 4.13(\mathrm{ddd}, J=2.0,6.0,12.0$, Hz), 3.8-3.75 (m, 1H), 3.71-3.66 (m, 1H), 3.66 (dd, $J=2.5,8.5, \mathrm{~Hz}), 2.89$ (dd, $J=2.5$, $16.0 \mathrm{~Hz}, 1 \mathrm{H}), 2.67(\mathrm{dd}, J=12.0,16.5 \mathrm{~Hz}, 1 \mathrm{H}), 2.13-2.06(\mathrm{~m}, 2 \mathrm{H}), 2.09$ (s, $6 \mathrm{H}), 2.03$ (s, $3 \mathrm{H}), 1.87-1.71(\mathrm{~m}, 3 \mathrm{H}), 1.65-1.58(\mathrm{~m}, 2 \mathrm{H}), 1.56(\mathrm{~s}, 3 \mathrm{H}), 1.35-1.25(\mathrm{~m}, 6 \mathrm{H}), 1.15(\mathrm{~d}, J=$ $7.0 \mathrm{~Hz}, 3 \mathrm{H}), 1.12-1.09$ (m, $36 \mathrm{H}), 0.91$ (s, $3 \mathrm{H}), 0.90$ (s, 9H), 0.84 (s, 3H), $0.11(\mathrm{~s}, 3 \mathrm{H})$, $0.08(\mathrm{~s}, 3 \mathrm{H}) ;{ }^{13} \mathrm{C} \mathrm{NMR}\left(\mathrm{CDCl}_{3}\right) \delta 171.0,163.0,159.1,157.7,141.1,118.4,109.7,77.9$, 74.8, 73.8, 72.0, 60.0, 43.1, 40.0, 36.3, 32.7, 30.2 , 26.4, 21.4, 21.3, 20.8, 19.6, 18.7, 18.2, 13.5, 13.3, 11.9, 9.6, -3.6; MS (ES) calculated for $\mathrm{C}_{49} \mathrm{H}_{91} \mathrm{O}_{10} \mathrm{Si}_{3}[\mathrm{M}+\mathrm{H}]^{+}$: 923.59, found 923.65; $\mathrm{C}_{49} \mathrm{H}_{90} \mathrm{NaO}_{10} \mathrm{Si}_{3}[\mathrm{M}+\mathrm{Na}]^{+}$: 945.57, found 945.62.

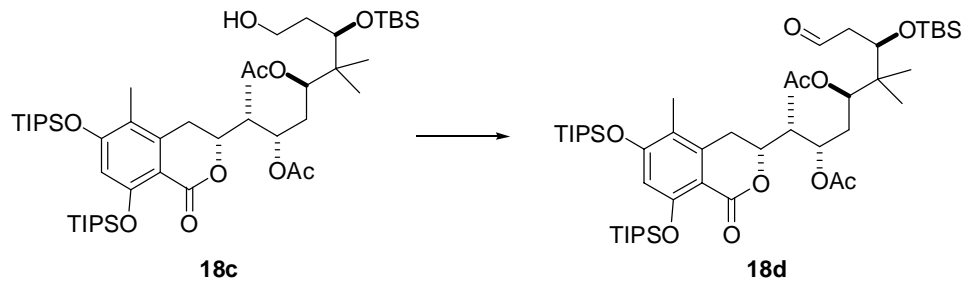

To a solution of compound 18c $(1.15 \mathrm{~g}, 1.24 \mathrm{mmol})$ in DCM $(20 \mathrm{ml})$ was added DMP (790 mg, $1.86 \mathrm{mmol}$ ) at $0{ }^{\circ} \mathrm{C}$. The reaction mixture was allowed to stir at $\mathrm{rt}$ for 2 hours. After the reaction is complete, it was cooled to $0{ }^{\circ} \mathrm{C}$ and aq. $\mathrm{Na}_{2} \mathrm{~S}_{2} \mathrm{O}_{3}(10 \mathrm{ml})$ was added. The mixture was stirred at rt for 15 minutes before being diluted with EtOAc, washed with aq. $\mathrm{NaHCO}_{3}$, brine, and dried over $\mathrm{Na}_{2} \mathrm{SO}_{4}$. The residue was purified by $\mathrm{FC}(\mathrm{E} / \mathrm{H}$ $1 / 10)$ to give the aldehyde $\mathbf{1 8 d}(1.08 \mathrm{~g}, 95 \%)$ as a colorless oil. ${ }^{1} \mathrm{HNMR}\left(\mathrm{CDCl}_{3}\right) \delta 9.86$ (s, 1H), 6.29 (s, 1H), 5.09-5.06 (m, 1H), 4.89 (dd, $J=2.0,10.0 \mathrm{~Hz}, 1 \mathrm{H}), 4.15$ (dd, $J=4.0$, $5.5 \mathrm{~Hz}), 4.07$ (ddd, $J=2.5,7.0,12.5 \mathrm{~Hz}, 1 \mathrm{H}), 2.93(\mathrm{dd}, J=2.5,16.0 \mathrm{~Hz}, 1 \mathrm{H}), 2.77$ (ddd, 
$J=1.0,4.0,18.0 \mathrm{~Hz}, 1 \mathrm{H}), 2.68-2.61(\mathrm{~m}, 2 \mathrm{H}), 2.10(\mathrm{~s}, 3 \mathrm{H}), 2.08(\mathrm{~s}, 3 \mathrm{H}), 2.02(\mathrm{~s}, 3 \mathrm{H})$, 2.08-2.06 (m, 1H), 1.81-1.75 (m, 1H), 1.35-1.25 (m, 6H), 1.15 (d, J = 7.0 Hz, 3H), 1.131.10 (m, $36 \mathrm{H}), 0.88$ (s, $3 \mathrm{H}), 0.88$ (s, 3H), 0.87 (s, 9H), 0.08 (s, 3H), $0.00(\mathrm{~s}, 3 \mathrm{H}) ;{ }^{13} \mathrm{C}$ NMR $\left(\mathrm{CDCl}_{3}\right) \delta 201.3,170.9,162.9,159.0,157.7,141.1,118.3,109.7,78.0,74.4,71.7$, $71.3,48.7,42.8,39.8,33.3,30.4,26.2,21.4,21.3,20.6,20.2,18.4,18.2,13.5,13.3,11.9$, 9.1, $-3.8,-4.6$.
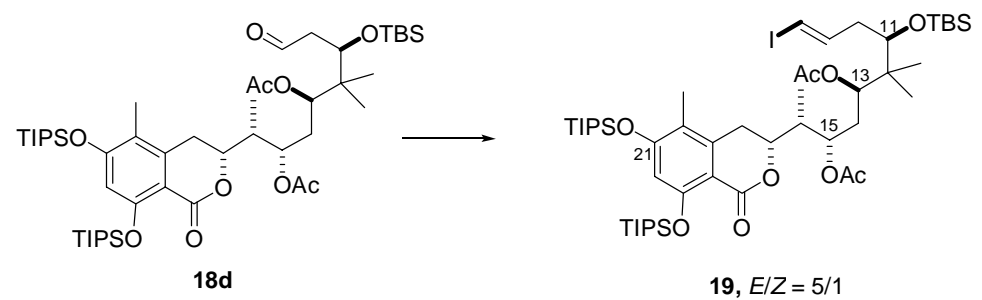

An aluminum foil wrapped round bottom flask containing $\mathrm{CrCl}_{2}(2.28 \mathrm{~g}, 18.6 \mathrm{mmol})$ was flamed dried under high vacuum and then cooled to room temperature. The adapter was quickly replaced with a rubber stopper and an argon balloon was place on the top. Aldehyde 18d (1.14 g, $1.24 \mathrm{mmol})$ was azeotropically dried with toluene three times before putting under high vacuum. To a suspension of $\mathrm{CrCl}_{2}(2.28 \mathrm{~g}, 18.6 \mathrm{mmol})$ in anhydrous THF $(20 \mathrm{ml})$ was added a solution of aldehyde $18 \mathrm{~d}(1.14 \mathrm{~g}, 1.24 \mathrm{mmol})$ and $\mathrm{CHI}_{3}(2.44 \mathrm{~g}, 6.2 \mathrm{mmol})$ in THF (20 ml plus $10 \mathrm{ml}$ washing) at $0{ }^{\circ} \mathrm{C}$. Color should turn to reddish brown after 10 to 15 minutes. The reaction mixture was allowed to stir at $\mathrm{rt}$ overnight before it was diluted with EtOAc and water was added. The mixture was extracted with EtOAc, washed with brine and dried over $\mathrm{Na}_{2} \mathrm{SO}_{4}$. After concentration the residue was purified by $\mathrm{FC}(\mathrm{E} / \mathrm{H} 1 / 20$ to $1 / 10)$ to give the vinyl iodide 19 (1.17 g, $90 \%)$ as a light yellow oil as a mixture of $E / Z$ isomers $(5 / 1) .{ }^{1} \mathrm{HNMR}\left(\mathrm{CDCl}_{3}\right) \delta 6.52-3.46(\mathrm{~m}$, $1 \mathrm{H}), 6.28(\mathrm{~s}, 1 \mathrm{H}), 6.06(\mathrm{~d}, J=14.5 \mathrm{~Hz}, 1 \mathrm{H}), 5.09-5.05(\mathrm{~m}, 1 \mathrm{H}), 4.91(\mathrm{dd}, J=1.5,10.5$ $\mathrm{Hz}, 1 \mathrm{H}$ ), 4.07 (ddd, $J=2.5,6.5,12.0 \mathrm{~Hz}, 1 \mathrm{H}$ ), 3.50 (dd, $J=4.0,7.0 \mathrm{hz}, 1 \mathrm{H}), 2.90$ (dd, $J=$ 2.0, $16.0 \mathrm{~Hz}, 1 \mathrm{H}), 2.63(\mathrm{dd}, J=12.0,16.0 \mathrm{~Hz}, 1 \mathrm{H}), 2.38-2.32(\mathrm{~m}, 1 \mathrm{H}), 2.25-2.19$ (m, $1 \mathrm{H}), 2.12-2.10(\mathrm{~m}, 1 \mathrm{H}), 2.08(\mathrm{~s}, 3 \mathrm{H}), 2.07(\mathrm{~s}, 3 \mathrm{H}), 2.02(\mathrm{~s}, 3 \mathrm{H}), 1.99-1.96(\mathrm{~m}, 1 \mathrm{H}), 1.78-$ $1.72(\mathrm{~m}, 1 \mathrm{H}), 1.34-1.24(\mathrm{~m}, 6 \mathrm{H}), 1.15(\mathrm{~d}, J=7.0 \mathrm{hz}, 3 \mathrm{H}), 1.12-1.09$ (m, $36 \mathrm{H}), 0.89$ (s, 9H), 0.88 (s, 3H), 0.85 (s, 3H), 0.06 (s, 3H), 0.05 (s, 3H); ${ }^{13} \mathrm{C} \mathrm{NMR}\left(\mathrm{CDCl}_{3}\right) \delta$ 171.0, 170.7, 162.8, 159.0, 157.7, 144.4, 141.0, 139.1, 118.3, 109.8, 109.7, 83.7, 78.1, 77.9, 77.1, 76.3, 74.4, 71.6, 43.4, 43.2, 40.2, 39.5, 32.7, 30.4, 26.3, 21.4, 21.3, 21.1, 20.9, 20.1, 
18.5, 18.2, 18.1, 13.5, 13.3, 11.9, 9.2, -3.1, -3.9; MS (ES) calculated for $\mathrm{C}_{50} \mathrm{H}_{90} \mathrm{IO}_{9} \mathrm{Si}_{3}[\mathrm{M}$ $+\mathrm{H}]^{+}: 1045.50$, found 1045.72 .

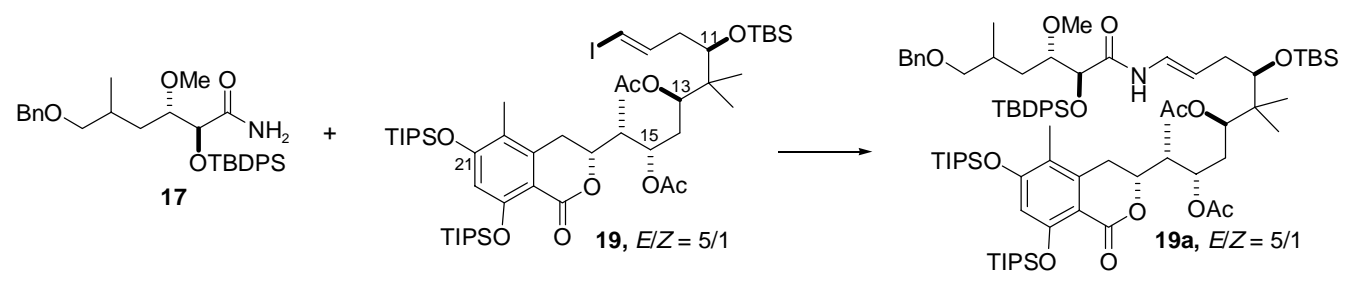

To an oven dried sealed tube was charged amide 17 (500 mg, $0.99 \mathrm{mmol}$ ), CuI (31 mg, $0.16 \mathrm{mmol}$ ), and $\mathrm{Cs}_{2} \mathrm{CO}_{3}(326 \mathrm{mg}, 1 \mathrm{mmol})$ sequentially. Dimethylethylenediamine ( $0.035 \mathrm{ml}, 0.32 \mathrm{mmol}$ ) was then added followed by a solution of vinyl iodide 19 (346 $\mathrm{mg}$, $0.33 \mathrm{mmol}$ ) in toluene under argon. The tube was filled with argon and quickly capped and sealed. The reaction was stirred vigorously at $70{ }^{\circ} \mathrm{C}$ for 20 hurs. After the reaction was cooled to rt, it was diluted with EtOAc and filtered through a short pad of silica gel and washed with EtOAc. The combined organic solution was concentrated and purified by FC (A/H 1/20 to 1/10) to give the enamide 19a (450 mg, 95\%) as an oil. The excess amide was recovered. For characterization purpose, a small amount of $E / Z$ mixture was separated. $E$ isomer: ${ }^{1} \mathrm{HNMR}\left(\mathrm{CDCl}_{3}\right) \delta 8.18(\mathrm{~d}, J=11.0 \mathrm{~Hz}, 1 \mathrm{H}), 7.69-7.30(\mathrm{~m}, 15 \mathrm{H})$, $6.62(\mathrm{dd}, J=12.0,13.5 \mathrm{~Hz}, 1 \mathrm{H}), 6.29(\mathrm{~s}, 1 \mathrm{H}), 5.10(\mathrm{br}, 1 \mathrm{H}), 5.03(\mathrm{~m}, 1 \mathrm{H}), 4.92(\mathrm{~d}, J=$ $9.0 \mathrm{~Hz}, 1 \mathrm{H}), 4.47$ (s, 1H), $4.45(\mathrm{dd}, J=5.0,10.0 \mathrm{~Hz}, 1 \mathrm{H}), 4.37$ (dd, $J=1.5,19.5 \mathrm{~Hz}, 1 \mathrm{H})$, 4.07 (ddd, $J=2.5,6.5,12.5 \mathrm{~Hz}, 1 \mathrm{H}), 3.42(\mathrm{~m}, 2 \mathrm{H}), 3.27(\mathrm{~m}, 1 \mathrm{H}), 3.19-3.08(\mathrm{~m}, 4 \mathrm{H}), 2.92$ (dd, $J=2.5,16.5 \mathrm{hz}, 1 \mathrm{H}), 2.63(\mathrm{dd}, J=12.5,16.5 \mathrm{~Hz}, 1 \mathrm{H}), 2.30(\mathrm{~m}, 1 \mathrm{H}), 2.15(\mathrm{~m}, 2 \mathrm{H})$, 2.12-1.72 (m, $13 \mathrm{H}), 1.60(\mathrm{~m}, 1 \mathrm{H}), 1.35-1.25(\mathrm{~m}, 8 \mathrm{H}), 1.18-1.10(\mathrm{~m}, 49 \mathrm{H}), 0.90-0.80$ (m, $18 \mathrm{H}), 0.02(\mathrm{~s}, 3 \mathrm{H}),-0.04(\mathrm{~s}, 3 \mathrm{H}) ;{ }^{13} \mathrm{C} \mathrm{NMR}\left(\mathrm{CDCl}_{3}\right) \delta 171.1,170.8,168.4,163.0,159.0$, 157.7, 141.2, 136.3, 135.9, 130.5, 128.5, 128.3, 128.1, 127.8, 127.7, 127.6, 123.1, 118.4, 110.0, 109.7, 82.1, 78.2, 76.4, 75.6, 74.7, 73.1, 73.0, 71.8, 43.3, 39.4, 33.9, 30.5, 27.3, 26.4, 21.5, 21.4, 21.1, 20.4, 18.6, 18.3, 18.2, 16.9, 13.5, 13.4, 11.9, 9.1, -2.8, -3.9; MS (FAB) calculated for $\mathrm{C}_{81} \mathrm{H}_{129} \mathrm{NNaO}_{13} \mathrm{Si}_{4}[\mathrm{M}+\mathrm{Na}]^{+}: 1458.8439$, found 1458.8439 .

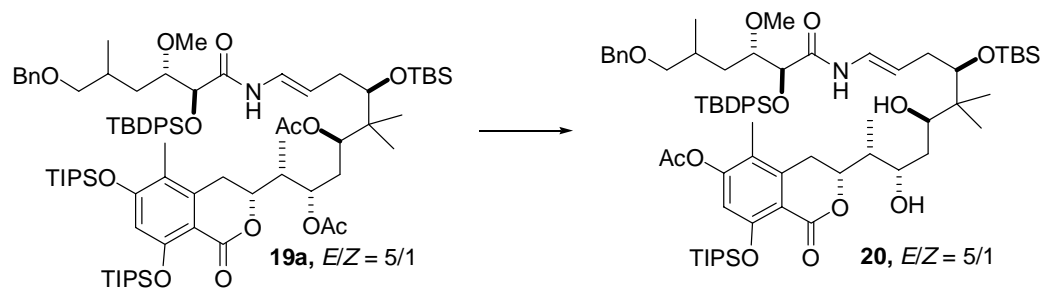


Compound 19a (450 mg, $0.31 \mathrm{mmol}$ ) was treated with a $\mathrm{NaOMe}$ solution in $\mathrm{MeOH}$ at $\mathrm{rt}$ for 5 hours before it was quenched by water. The mixture was then extracted with EtOAc thoroughly. The organic layer was washed with brine, dried over $\mathrm{Na}_{2} \mathrm{SO}_{4}$ and concentrated. The crude was dissolved in DCM. Pyridine, DMAP and then $\mathrm{Ac}_{2} \mathrm{O}$ were added sequentially at $0{ }^{\circ} \mathrm{C}$. The reaction was allowed to stir at $0{ }^{\circ} \mathrm{C}$ for 1 hour before it was quenched with aq. $\mathrm{NH}_{4} \mathrm{Cl}$. The aq. layer was extracted with EtOAc. Combined organic layers were washed with $\mathrm{NH}_{4} \mathrm{Cl}$ and brine. After being dried and concentrated, the residue was purified by FC (Acetone/Hexane 1/20 to 1/6) to give 20 as a mixture (368 $\mathrm{mg}, 95 \%)$ of four isomers (E/Z-isomers (5:1) were separable on PTLC, hence a small part was separated for characterization purpose). The mixture was carried on to the next reaction. NMR data of the major $E$-isomer (NMR spectrum of the $Z$-isomer is attached): ${ }^{1} \mathrm{HNMR}\left(\mathrm{CDCl}_{3}\right) \delta 8.19(\mathrm{~d}, J=10.5 \mathrm{~Hz}, 1 \mathrm{H}), 7.69-7.26(\mathrm{~m}, 15 \mathrm{H}), 6.65(\mathrm{dd}, J=11.5$, $13.5 \mathrm{~Hz}, 1 \mathrm{H}), 6.53(\mathrm{~s}, 1 \mathrm{H}), 5.00(\mathrm{~m}, 1 \mathrm{H}), 4.86(\mathrm{~s}, 1 \mathrm{H}), 4.47(\mathrm{~s}, 1 \mathrm{H}), 4.43-4.36(\mathrm{~m}, 2 \mathrm{H})$, $4.26(\mathrm{~s}, 1 \mathrm{H}), 4.15(\mathrm{~d}, J=9.0 \mathrm{~Hz}, 1 \mathrm{H}), 4.06(\mathrm{~d}, J=10.5 \mathrm{~Hz}, 1 \mathrm{H}), 3.43(\mathrm{~m}, 2 \mathrm{H}), 3.26(\mathrm{~m}$, $1 \mathrm{H}), 3.18(\mathrm{~m}, 1 \mathrm{H}), 3.14-3.08(\mathrm{~m}, 4 \mathrm{H}), 3.03(\mathrm{~d}, J=16.5 \mathrm{~Hz}, 1 \mathrm{H}), 2.86(\mathrm{dd}, J=12.5,16.0$ Hz, 1H), $2.42(\mathrm{~m}, 1 \mathrm{H}), 2.33(\mathrm{~s}, 3 \mathrm{H}), 2.26(\mathrm{~m}, 1 \mathrm{H}), 2.05-1.99(\mathrm{~m}, 4 \mathrm{H}), 1.88(\mathrm{~m}, 2 \mathrm{H}), 1.62$ $(\mathrm{m}, 1 \mathrm{H}), 1.40-1.30(\mathrm{~m}, 4 \mathrm{H}), 1.15-1.10(\mathrm{~m}, 29 \mathrm{H}), 1.01$ (s, 3H), 0.89 (s, 9H), 0.92 (d, $J=$ $7.0 \mathrm{~Hz}, 3 \mathrm{H}$, isomer I), 0.86 (d, $J=7.0 \mathrm{~Hz}, 3 \mathrm{H}$, isomer II), 0.82 (s, 3H), 0.14 (s, 3H), 0.05 (s, 3H); ${ }^{13} \mathrm{C}$ NMR $\left(\mathrm{CDCl}_{3}\right) \delta 168.6,168.4,168.3,162.7,157.0,153.0,142.2,136.2$, 135.8, 130.5, 130.4, 128.4, 128.2, 128.0, 127.6, 127.5, 115.0, 113.5, 84.9, 81.6, 78.8, 77.8, 76.2, 75.5, 73.0, 72.9, 72.4, 43.0, 34.9, 33.1, 30.3, 30.0, 29.7, 27.2, 26.2, 23.7, 21.1, 19.7, 18.4, 18.3, 18.2, 16.8, 13.3, 12.0, 9.99, -3.3, -3.9; MS (FAB) calculated for $\mathrm{C}_{70} \mathrm{H}_{107} \mathrm{NNaO}_{12} \mathrm{Si}_{3}[\mathrm{M}+\mathrm{Na}]^{+}$1260.6999, found 1260.7005 .
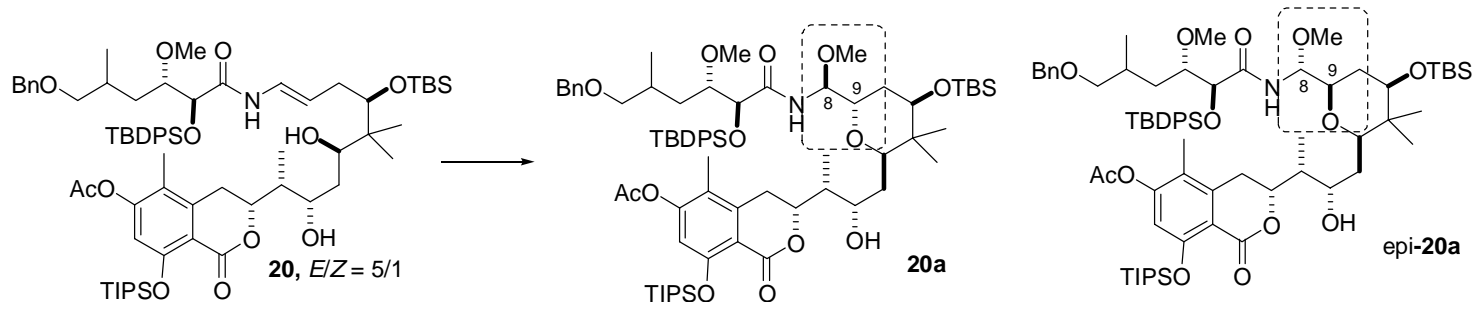

Under an atmosphere of argon, to a solution of compound 20 (300 mg, $0.24 \mathrm{mmol}$ ) in HFI (4 ml) at $0{ }^{\circ} \mathrm{C}$ was added $\mathrm{MeOH}(0.2 \mathrm{ml})$ and then a solution of DIB $(320 \mathrm{mg}, 0.96$ $\mathrm{mmol})$ in HFI ( $2 \mathrm{ml})$ dropwise. The reaction mixture was stirred at $\mathrm{rt}$ for 70 hours before it was diluted with EtOAc and filtered through a short pad of silica gel. The filtrate was 
concentrated and then purified by FC (acetone/hexane $1 / 10$ to $1 / 2$ to give two crude fraction which was repurified with EtOAc/toluene 1/10 to 1/2) to give 20a and epi-20a. (90 mg each, $60 \%$ combined). A mixture of other possible isomers $(\sim 12 \%)$ was also obtained which was not fully characterized. 20a: ${ }^{1} \mathrm{HNMR}\left(\mathrm{CDCl}_{3}\right) \delta 7.69-7.24(\mathrm{~m}, 15 \mathrm{H})$, 6.52, 6.51 (s, 1H), 5.19 (m, 1H), 4.43-4.38 (m, 3H), 4.26 (br, 1H), 4.08 (br, 1H), 3.97 (br, $1 \mathrm{H}), 3.72,3.70(\mathrm{~s}, 1 \mathrm{H}), 3.52-3.48(\mathrm{~m}, 2 \mathrm{H}), 3.34(\mathrm{~s}, 3 \mathrm{H}), 3.33(\mathrm{~s}, 3 \mathrm{H}), 3.29(\mathrm{~m}, 1 \mathrm{H}), 3.22-$ 3.00 (m, 6h), 2.92-2.85 (m, 1H), 2.31 (s, 3H), 2.05 (s, 3H), 2.04 (s, 3H), 1.84-1.44 (m, $3 \mathrm{H}), 1.37-1.31(\mathrm{~m}, 3 \mathrm{H}), 1.13-1.10(\mathrm{~m}, 30 \mathrm{H}), 0.90(\mathrm{~s}, 9 \mathrm{H}), 0.80(\mathrm{~s}, 3 \mathrm{H}), 0.77(\mathrm{~s}, 3 \mathrm{H})$, $0.74,0.71(\mathrm{~d}, J=6.5 \mathrm{~Hz}, 3 \mathrm{H}), 0.04(\mathrm{~s}, 6 \mathrm{H}) ;{ }^{13} \mathrm{C} \mathrm{NMR}\left(\mathrm{CDCl}_{3}\right) \delta 172.4,172.2,168.5$, 162.7, 157.0, 152.9, 142.3, 136.3, 135.9, 132.8, 130.3, 130.1, 128.4, 128.0, 127.9, 127.6, $127.5,113.3,82.3,79.1,76.2,75.5,72.9,72.2,56.2,43.3,34.5,30.4,30.0,29.6,27.3$, 25.9, 21.0, 19.6, 18.1, 16.8, 14.3, 13.3, 12.0, 9.8, -4.4, -4.9. MS (FAB) calculated for $\mathrm{C}_{71} \mathrm{H}_{109} \mathrm{NO}_{13} \mathrm{NaSi}_{3}[\mathrm{M}+\mathrm{Na}]^{+} 1290.7104$, found 1290.7100; NMR spectrum of epi-20a is attached.
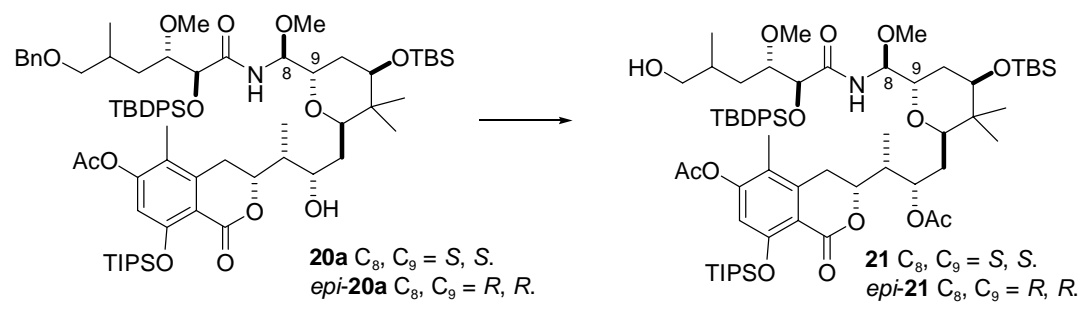

To a solution of compound 20a (48 $\mathrm{mg}, 0.038 \mathrm{mmol})$ in DCM (1 ml) was added pyridine $(0.30 \mathrm{ml}), \mathrm{Ac}_{2} \mathrm{O}(0.15 \mathrm{ml})$ and DMAP $(2.5 \mathrm{mg}, 0.02 \mathrm{mmol})$ sequentially. The reaction mixture was stirred at $\mathrm{rt}$ overnight before being quenched with aq. $\mathrm{NH}_{4} \mathrm{Cl}$ and the aq. layer was extracted with EtOAc thoroughly. The combine organic layers were washed with brine, dried over $\mathrm{Na}_{2} \mathrm{SO}_{4}$ and concentrated. The residue was passed through a short silica gel plug then directly put to the next step. To a solution of the acetate compound (20 mg, $0.015 \mathrm{mmol})$ in $\mathrm{MeOH}(2 \mathrm{ml})$ was charged $\mathrm{Pd} / \mathrm{C}(5 \mathrm{mg})$ and the flask was sealed with rubber stopper. The inner atmosphere was exchanged three times with hydrogen before it was allowed to stir under $\mathrm{H}_{2}$ (double layer $\mathrm{H}_{2}$ balloon) overnight. The reaction mixture was filtered through a celite pad followed by washing with $\mathrm{MeOH}$ twice. The combined organic solution was concentrated and purified by FC to give the alcohol 21 (17.3 mg, 95\%). epi-21 was obtained in the same sequence and two isomers (epi-21-I, epi-21-II, spectrum attached) could be separated by PTLC. 21: ${ }^{1} \mathrm{HNMR}\left(\mathrm{CDCl}_{3}\right) \delta$ 7.68- 
$7.33(\mathrm{~m}, 10 \mathrm{H}), 6.52$ (s, 1H), $5.39(\mathrm{~m}, 1 \mathrm{H}), 5.07$ (dm, $J=10.0 \mathrm{~Hz}, 1 \mathrm{H}), 4.49$ (dd, $J=2.0$, $10.0 \mathrm{~Hz}, 1 \mathrm{H}), 4.19$ (br, 1H), $4.11(\mathrm{~m}, 1 \mathrm{H}), 3.55$ (br, 1H), 3.39, 3.38 (s, 3H), 3.34 (dm, J= $12.5 \mathrm{~Hz}, 1 \mathrm{H}), 3.23$ (m, 2H), 3.13 (dm, $J=16.5 \mathrm{~Hz}, 1 \mathrm{H}), 2.99,2.98$ (s, 3H), 2.68-2.61 (m, 2H), 2.32 (s, 3H), 2.14 (m, 1H), 2.09 (m, 1H), 2.02 (s, 3H), 1.98, 1.97 (s, 3H), 1.77-1.39 (m, 6H), 1.37-1.30 (m, 3H), $1.18(\mathrm{~d}, J=7.0 \mathrm{~Hz}, 3 \mathrm{H}), 1.13-1.11(\mathrm{~m}, 29 \mathrm{H}), 0.88(\mathrm{~s}, 9 \mathrm{H})$, 0.82 (s, 3H), 0.77, 0.76 (s, 3H), 0.66, 0.61 (d, J=7.0 Hz, 3H), 0.05 (s, 3H), 0.04 (s, 3H); ${ }^{13} \mathrm{C} \mathrm{NMR}\left(\mathrm{CDCl}_{3}\right) \delta 172.3,172.1,170.5,168.7,162.3,157.0,153.0,141.8,136.3,135.7$, $132.9,132.8,130.4,130.3,130.1,128.0,127.9,119.6,119.5,114.6,113.4,83.0,82.5$, 81.7, 79.2, 71.4, 68.1, 67.3, 57.6, 56.5, 33.5, 32.4, 30.0, 27.3, 25.9, 21.3, 21.1, 19.6, 18.1, 18.0, 17.5, 17.2, 13.3, 11.9, 11.8, -4.4, -5.0; MS (FAB) calculated for $\mathrm{C}_{66} \mathrm{H}_{105} \mathrm{NO}_{14} \mathrm{Na} \mathrm{Si}_{3}$ $[\mathrm{M}+\mathrm{Na}]^{+}$1242.6741, found 1242.6745. epi-21-I: ${ }^{1} \mathrm{HNMR}\left(\mathrm{CDCl}_{3}\right) \delta 7.86(\mathrm{~d}, J=10.0$ $\mathrm{Hz}, 1 \mathrm{H}), 7.73-7.34(\mathrm{~m}, 10 \mathrm{H}), 6.52(\mathrm{~s}, 1 \mathrm{H}), 5.34(\mathrm{~m}, 1 \mathrm{H}), 4.90(\mathrm{~d}, J=10.0 \mathrm{~Hz}, 1 \mathrm{H}), 4.47$, $(\mathrm{d}, J=1.5 \mathrm{~Hz}, 1 \mathrm{H}), 4.08(\mathrm{~m}, 1 \mathrm{H}), 3.65(\mathrm{dm}, J=10.5 \mathrm{~Hz}, 1 \mathrm{H}), 3.42(\mathrm{dd}, J=5.0,11.0 \mathrm{~Hz}$, $1 \mathrm{H}), 4.47$ (d, $J=1.5 \mathrm{~Hz}, 1 \mathrm{H}), 4.08(\mathrm{~m}, 1 \mathrm{H}), 3.65(\mathrm{dm}, J=10.5 \mathrm{~Hz}, 1 \mathrm{H}), 3.42$ (dd, $J=5.0$, $11.0 \mathrm{~Hz}, 1 \mathrm{H}), 3.23$ (s, 3H), 3.27-3.10 (m, 4H), 3.00-2.95 (m, 1H), 2.97 (s, 3H), 2.66 (dd, $J=12.0,16.0 \mathrm{~Hz}, 1 \mathrm{H}), 2.34$ (s, 3H), 2.30 (m, 1H), 2.01 (s, 3H), 1.97 (s, 3H), 2.03-1.94 (m, 2H), 1.78-1.42 (m, 8H), 1.36-1.28 (m, 3H), 1.16-1.10 (m, 36 H), 0.92 (s, 3H), 0.87 (s, $9 \mathrm{H}), 0.64(\mathrm{~d}, J=6.5 \mathrm{~Hz}, 3 \mathrm{H}), 0.05(\mathrm{~s}, 3 \mathrm{H}), 0.01(\mathrm{~s}, 3 \mathrm{H}) . . \mathrm{MS}(\mathrm{FAB})$ calculated for $\mathrm{C}_{66} \mathrm{H}_{105} \mathrm{NO}_{14} \mathrm{NaSi}_{3}[\mathrm{M}+\mathrm{Na}]^{+}$1242.6741, found 1242.6745 .
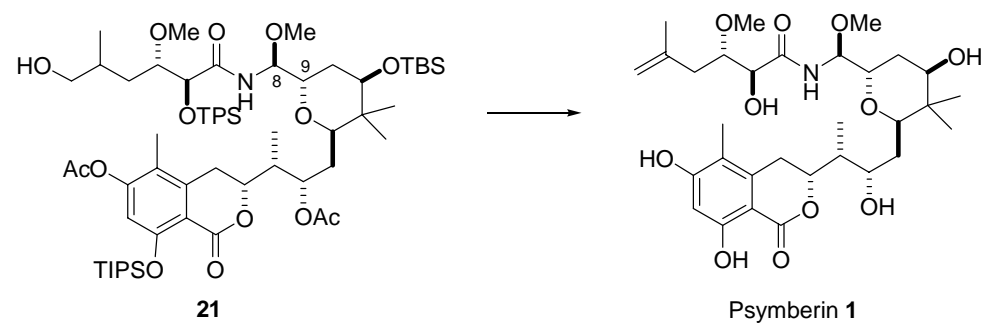

Substrate 21 was azeotropically dried with toluene three times before use. To a solution of compound 21 (25 mg, $0.02 \mathrm{mmol})$ in anhydrous THF (1 ml) was charged with $o$ nitrophenylselenocyanate $(18 \mathrm{mg}, 0.08 \mathrm{mmol})$ followed by $\mathrm{Bu}_{3} \mathrm{P}(0.025 \mathrm{ml}, 0.1 \mathrm{mmol})$. The reaction mixture was allowed to stir at rt for 2 hours before it was quenched by adding $\mathrm{MeOH}(0.5 \mathrm{ml})$. The mixture was stirred for an additional 15 minutes before it was concentrated. The residue was purified by FC to give the desired selenium product. 
(A little partial phenol acetate and TIPS deprotected compounds were observed which were converted to the final product in the same sequence.) To a solution of the selenium compounds was added THF $(2 \mathrm{ml})$ and $30 \%$ aq. $\mathrm{H}_{2} \mathrm{O}_{2}(0.2 \mathrm{ml})$ at $0{ }^{\circ} \mathrm{C}$. The reaction mixture was stirred at $\mathrm{rt}$ for 45 minutes and then at $50{ }^{\circ} \mathrm{C}$ for 1.5 hours before it was diluted with EtOAc and washed with $\mathrm{Na}_{2} \mathrm{SO}_{3}$ thoroughly. The organic phase was dried $\left(\mathrm{Na}_{2} \mathrm{SO}_{4}\right)$ and concentrated, the residue was dissolved in THF (1 ml) followed with addition of TBAF $(0.1 \mathrm{M}, 0.3 \mathrm{ml})$. The reaction mixture was stirred at $50{ }^{\circ} \mathrm{C}$ for 20 hours and TLC showed only one compound with bright UV. The reaction was quenched by addition of water $(1 \mathrm{ml})$ and diluted with EtOAc $(20 \mathrm{ml})$, the aqueous layer was separated and $\mathrm{pH}$ was adjusted to 6 with aq. $\mathrm{NaHSO}_{4}$ solution and then extracted with EtOAc thoroughly. Combined organic layers were washed with brine, dried $\left(\mathrm{Na}_{2} \mathrm{SO}_{4}\right)$ and concentrated. PTLC of the residue gave psymberin 1 ( $8.1 \mathrm{mg}, 67 \%$ over three steps) as a glass solid. $[\alpha]^{23}{ }_{\mathrm{D}}=+19.5(\mathrm{MeOH}, \mathrm{c}=0.2){ }^{1} \mathrm{HNMR}\left(\mathrm{CDCl}_{3}\right) \delta 11.13(\mathrm{~s}, 1 \mathrm{H}), 7.22(\mathrm{br}$, $1 \mathrm{H}), 7.12(\mathrm{~d}, J=10.0 \mathrm{~Hz}, 1 \mathrm{H}), 6.31(\mathrm{~s}, 1 \mathrm{H}), 5.44(\mathrm{dd}, J=9.0,10.0 \mathrm{~Hz}, 1 \mathrm{H}), 4.81(\mathrm{~s}, 1 \mathrm{H})$, 4.80 (s, 1H), 4.54 (ddd, $J=4.0,4.0,12.5 \mathrm{~Hz}, 1 \mathrm{H}), 4.43$ (dd, $J=2.5,3.0 \mathrm{~Hz}, 1 \mathrm{H}), 4.40$ (s, 1H), 4.17 (br, 1H), $3.94(\mathrm{dm}, J=8.5 \mathrm{~Hz}, 1 \mathrm{H}), 3.89(\mathrm{~m}, 1 \mathrm{H}), 3.75(\mathrm{~m}, 1 \mathrm{H}), 3.66(\mathrm{dd}, J=$ $4.0,10.5 \mathrm{~Hz}, 1 \mathrm{H}), 3.53$ ( d, $J=11.0 \mathrm{~Hz}, 1 \mathrm{H}), 3.38$ (s, $3 \mathrm{H}), 3.37$ (s, 3H), 2.87 (dd, $J=3.0$, $16.0 \mathrm{~Hz}, 1 \mathrm{H}), 2.80$ (dd, $J=12.5,16.5 \mathrm{~Hz}, 1 \mathrm{H}), 2.38(\mathrm{dd}, J=9.0,14.5 \mathrm{~Hz}, 1 \mathrm{H}), 2.18$ (dd, $J=4.5,14.5 \mathrm{~Hz}, 1 \mathrm{H}), 2.06(\mathrm{~m}, 1 \mathrm{H}), 2.01(\mathrm{~s}, 3 \mathrm{H}), 1.88(\mathrm{~m}, 1 \mathrm{H}), 1.80(\mathrm{~m}, 1 \mathrm{H}), 1.75(\mathrm{~s}$, $3 \mathrm{H}), 1.62(\mathrm{~m}, 1 \mathrm{H}), 1.09(\mathrm{~d}, J=7.5 \mathrm{~Hz}, 3 \mathrm{H}), 0.96(\mathrm{~s}, 3 \mathrm{H}), 0.91(\mathrm{~s}, 3 \mathrm{H}) ;{ }^{13} \mathrm{C} \mathrm{NMR}\left(\mathrm{CDCl}_{3}\right)$ $\delta$ 173.8, 170.7, 162.5, 161.4, 142.2, 139.9, 113.6, 113.3, 101.7, 101.5, 82.2, 80.7, 79.6, 78.6, 74.0, 73.3 , 71.6, 58.2, 56.5, 42.8, 39.0, 37.8, 32.3, 29.9, 28.6, 23.3, 22.9, 14.0, 10.7, 9.7; MS (ES) calculated for $\mathrm{C}_{31} \mathrm{H}_{47} \mathrm{O}_{11} \mathrm{Na}[\mathrm{M}+\mathrm{Na}]^{+}$632.30, found 632.23.

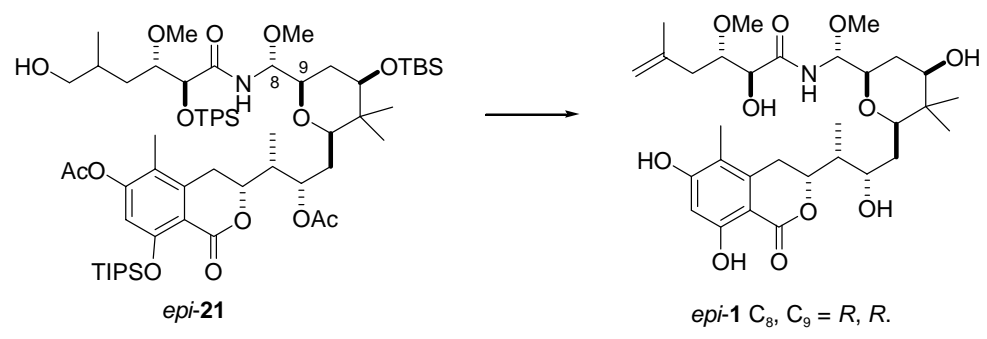

The same procedure for the synthesis of psymberin 1 was applied to compound epi-21. The only difference is that treatment with TBAF was not able to remove the acetate at 
$\mathrm{C}_{15}$. In this case, after removal of the silyl groups by TBAF, the crude was dissolved in $\mathrm{MeOH}(1 \mathrm{ml})$ and aq. $\mathrm{LiOH}(1 \mathrm{~N}, 0.2 \mathrm{ml})$. The reaction mixture was stirred at $\mathrm{rt}$ overnight before it was quenched with addition of water and EtOAc. The aq. layer was separated and $\mathrm{pH}$ was adjusted to 6 with aq. $\mathrm{NaHSO}_{4}$ solution, and then extracted with EtOAc thoroughly. Combined organic layers were washed with brine, dried and concentrated. PTLC of the residue gave epi-1 as a glass solid. $[\alpha]^{23} \mathrm{D}=-13.5(\mathrm{MeOH}, \mathrm{c}=0.1){ }^{1} \mathrm{HNMR}$ $\left(\mathrm{CDCl}_{3}\right) \delta 11.24(\mathrm{~s}, 1 \mathrm{H}), 7.37$ (d, $\left.J=9.5 \mathrm{~Hz}, 1 \mathrm{H}\right), 6.28(\mathrm{~s}, 1 \mathrm{H}), 5.04(\mathrm{dd}, J=2.5,10.0 \mathrm{~Hz}$, $1 \mathrm{H}), 4.83(\mathrm{~s}, 1 \mathrm{H}), 4.80(\mathrm{~s}, 1 \mathrm{H}), 4.56$ (ddd, $J=3.5,9.0,13.0 \mathrm{~Hz}, 1 \mathrm{H}), 4.36(\mathrm{~d}, J=4.0 \mathrm{~Hz}$, $1 \mathrm{H}), 4.12(\mathrm{~m}, 1 \mathrm{H}), 3.80(\mathrm{dm}, J=11.5 \mathrm{~Hz}, 1 \mathrm{H}), 3.73(\mathrm{~m}, 1 \mathrm{H}), 3.49(\mathrm{dd}, J=5.0,11.0 \mathrm{~Hz}$, $1 \mathrm{H}), 3.43(\mathrm{~s}, 3 \mathrm{H}), 3.35(\mathrm{~s}, 3 \mathrm{H}), 3.31(\mathrm{~d}, J=10.5 \mathrm{~Hz}, 1 \mathrm{H}), 2.95(\mathrm{dd}, J=4.0,16.5 \mathrm{~Hz}, 1 \mathrm{H})$, $2.90(\mathrm{dd}, J=12.0,16.5 \mathrm{~Hz}, 1 \mathrm{H}), 2.33(\mathrm{dd}, J=9.0,14.5 \mathrm{~Hz}, 1 \mathrm{H}), 2.20(\mathrm{~m}, 1 \mathrm{H}), 2.04$ (s, 3H), $1.95(\mathrm{~m}, 1 \mathrm{H}), 1.77(\mathrm{~s}, 3 \mathrm{H}), 1.73(\mathrm{~m}, 1 \mathrm{H}), 1.65(\mathrm{~m}, 1 \mathrm{H}), 1.44(\mathrm{~m}, 1 \mathrm{H}), 1.13(\mathrm{~d}, J=$ $7.5 \mathrm{~Hz}, 3 \mathrm{H}), 0.96$ (s, 3H), $0.88(\mathrm{~s}, 3 \mathrm{H}) ;{ }^{13} \mathrm{C} \mathrm{NMR}\left(\mathrm{CDCl}_{3}\right) \delta 172.8,170.8,162.5,160.9$, $142.1,140.3,113.5,101.5,86.5,81.3,80.8,80.0,74.8,73.0,71.3,57.9,56.5,42.8,39.4$, 37.5, 32.3, 32.2, 28.4, 23.0, 22.4, 12.7, 10.8, 9.9; MS (ES) calculated for $\mathrm{C}_{31} \mathrm{H}_{47} \mathrm{O}_{11} \mathrm{Na}$ $[\mathrm{M}+\mathrm{Na}]^{+} 632.30$, found 632.23 . 
Top spectrum: synthetic psymberin by De Brabander group (number from the original publication); Middle spectrum: natural psymberin; (Both spectra scanned from J. Am. Chem. Soc. 2005, 127, 11254 in supporting information. We thank Professor De Brabander for the permission.) Bottom spectrum: synthetic psymberin from this publication.
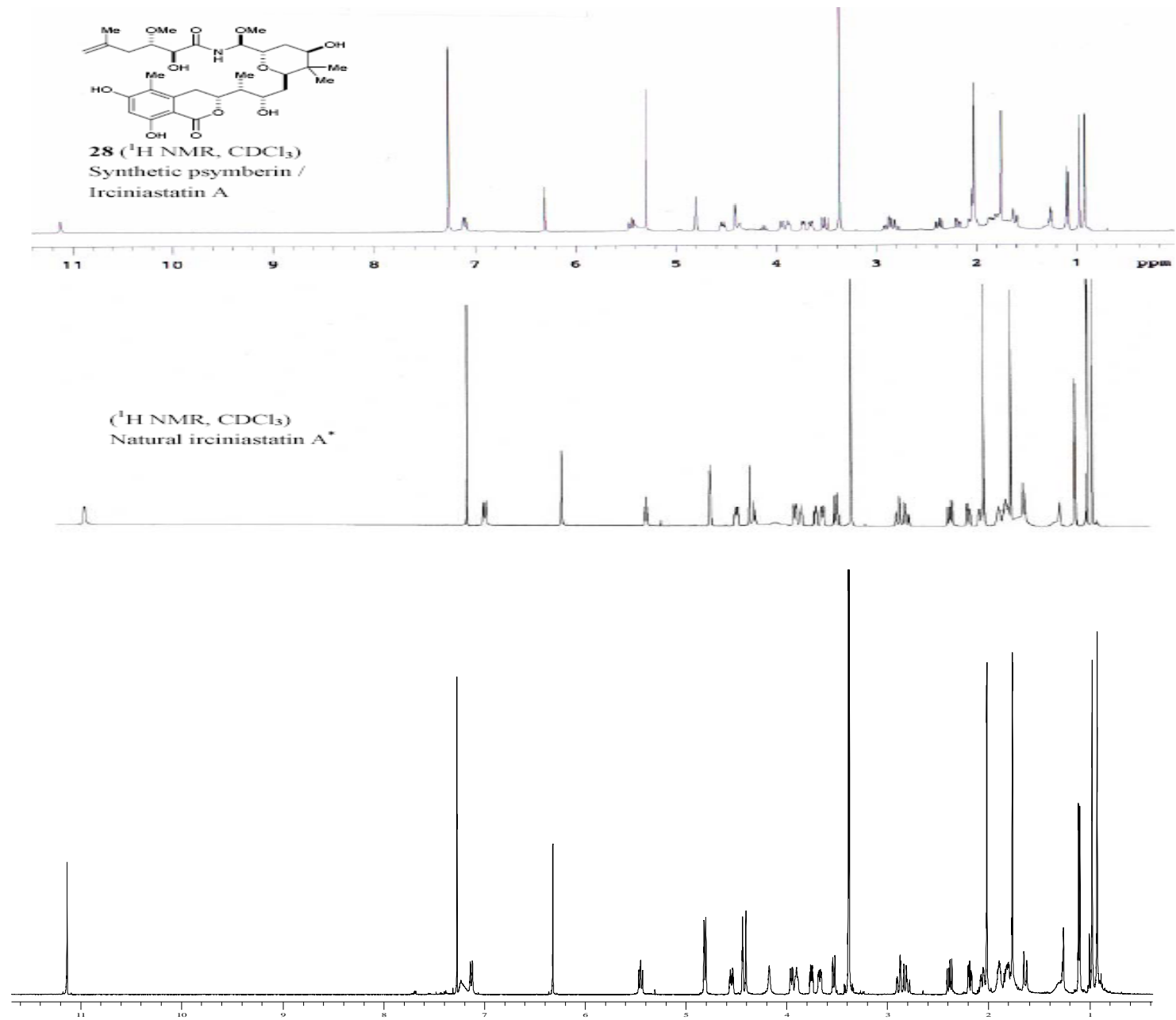

Synthetic Psymberin 1: (in $\mathrm{CDCl}_{3}$ ) 
Top spectrum: synthetic psymberin by De Brabander group (number from the original publication); Middle spectrum: natural psymberin; (Both spectra scanned from $J$. Am. Chem. Soc. 2005, 127, 11254 in supporting information. We thank Professor De Brabander for the permission.) Bottom spectrum: synthetic psymberin from this publication.
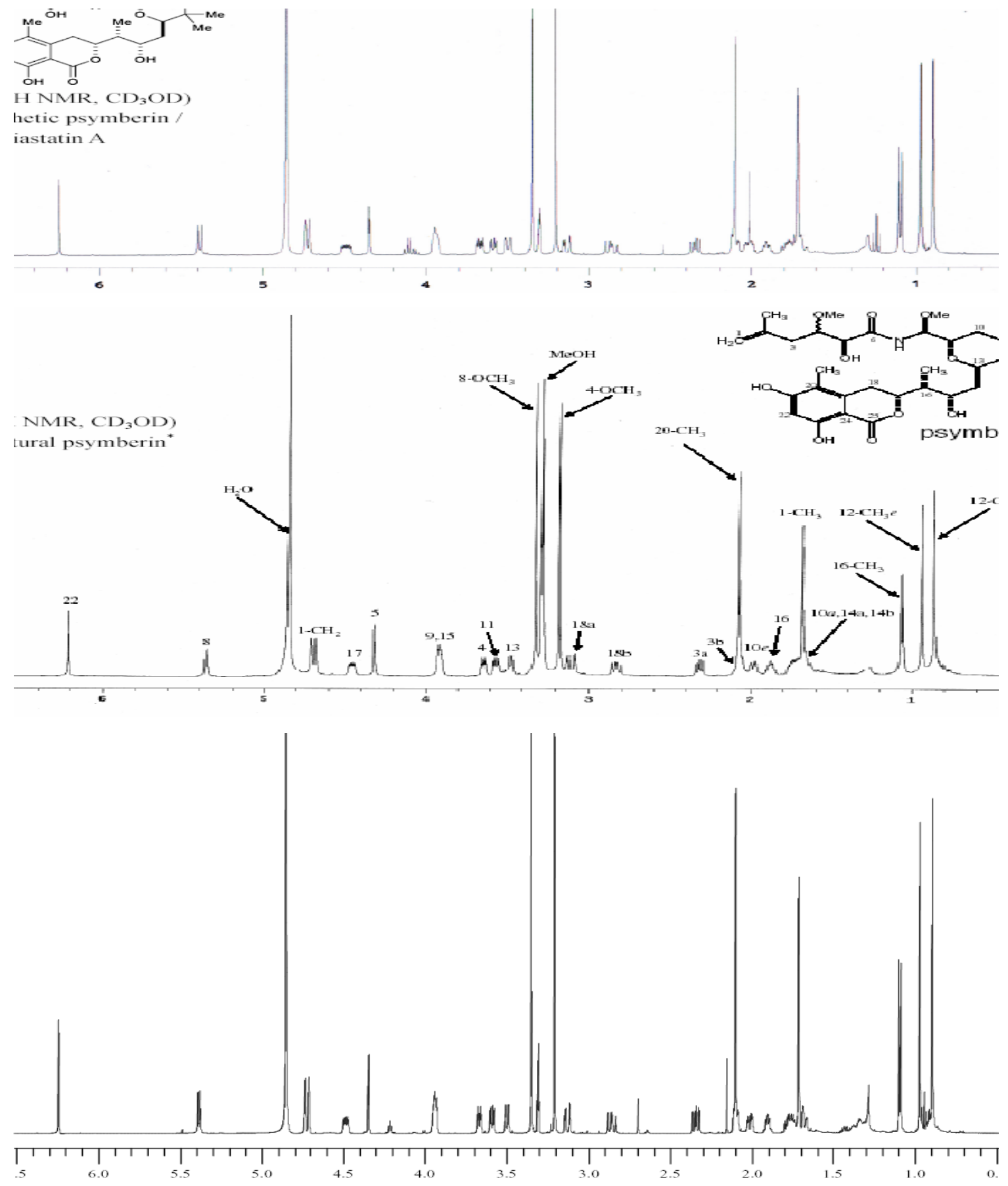

Synthetic Psymberin 1: (in $\mathrm{CD}_{3} \mathrm{OD}$ ) 
Top spectrum: synthetic psymberin by De Brabander group (number from the original publication); Middle spectrum: natural psymberin; (Both spectra scanned from J. Am. Chem. Soc. 2005, 127, 11254 in the supporting information. We thank Professor De Brabander for the permission.) Bottom spectrum: synthetic psymberin from this publication.

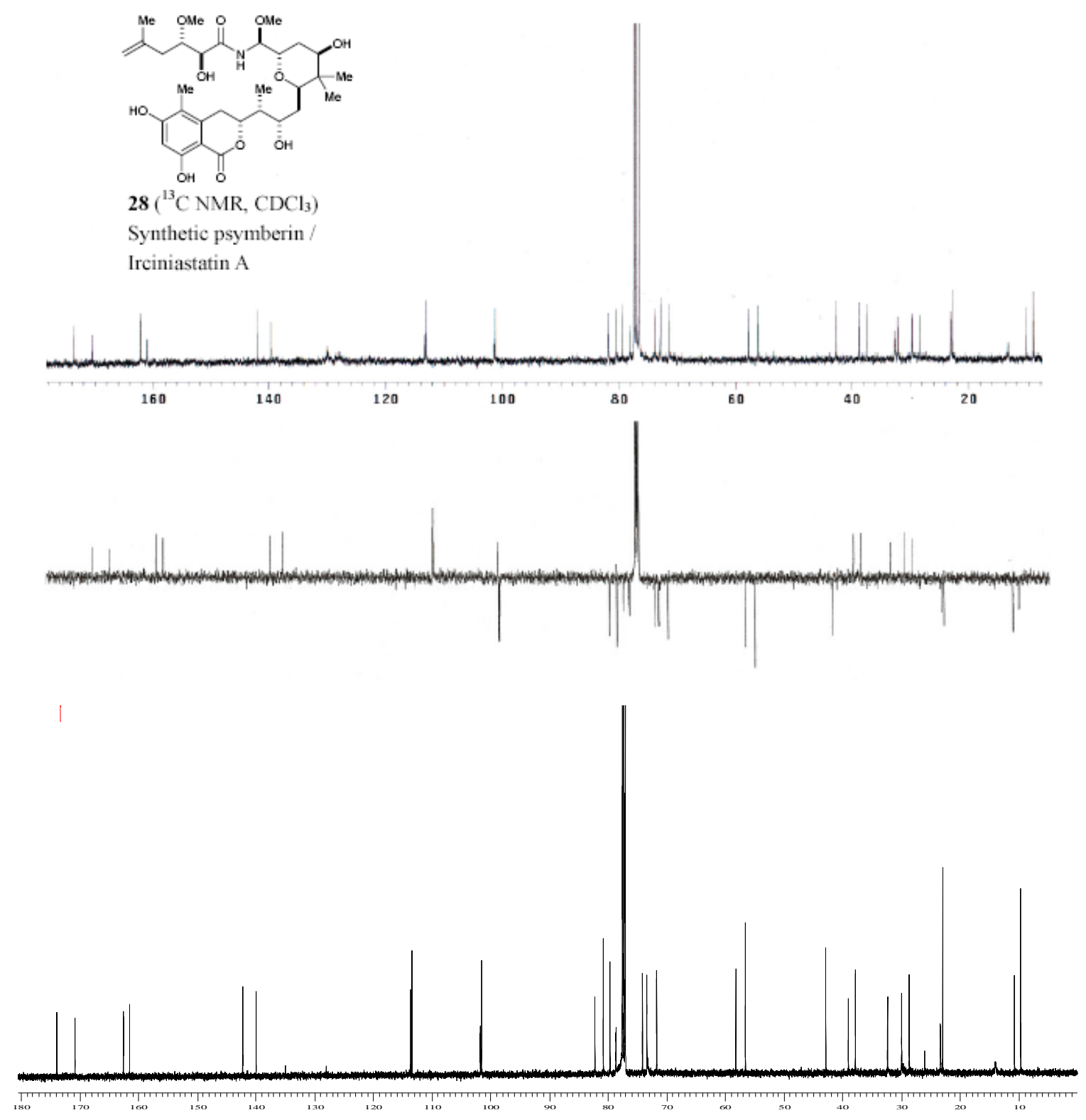

Synthetic Psymberin 1: (in $\mathrm{CDCl}_{3}$ ) 


\section{Structure determination of $e p i-1$}

Sample of epi-1 was analyzed by NMR. Proton, carbon, proton-proton and proton-carbon correlation NMR spectra were collected on a Varian $600 \mathrm{MHz}$ spectrometer at $25{ }^{\circ} \mathrm{C}$ in $\mathrm{CDCl}_{3}$. NMR spectra of epi-1 were consistent with the structure shown below (stereochemistry at C10 and $\mathrm{C} 12)$.
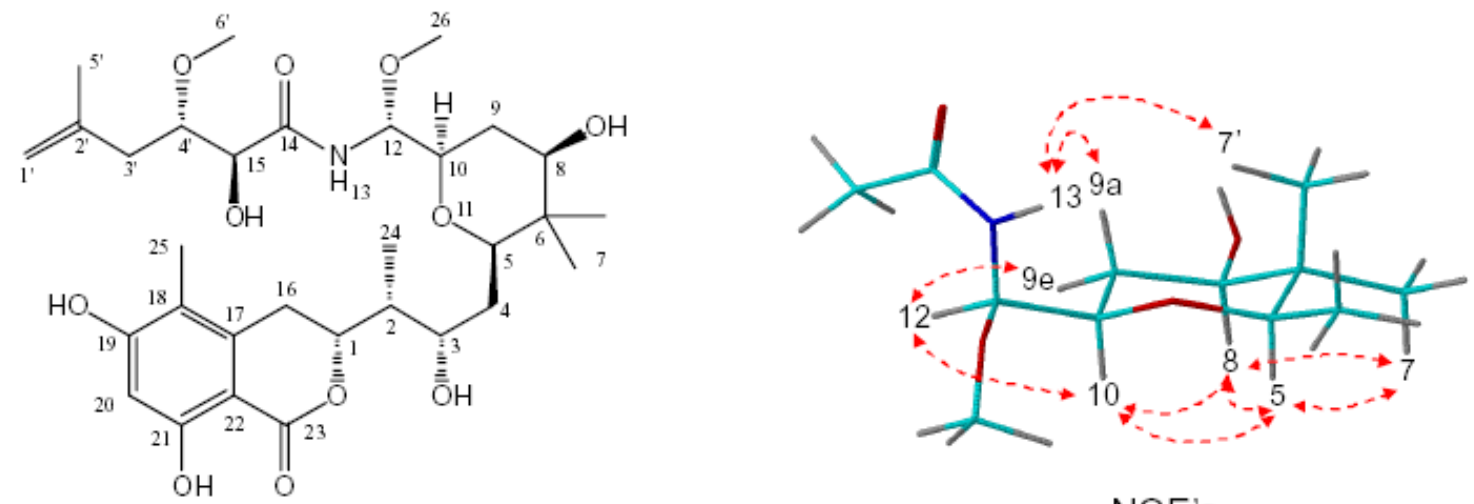

epi-1

NOE's

Proton and carbon resonances of epi-1 were assigned by using COSY, NOESY, HSQC and HMBC experiments. Relative stereochemistry of C10, C12 centers was defined based on protonproton couplings and NOE's. Thus, large coupling between H10 and H9a protons (11.9 Hz) showed axial orientation of the H10 proton. Small coupling between H10 and H12 protons (2.6 $\mathrm{Hz}$ ) showed their gauche orientation. NOE's between H10, H8 and H5 protons indicated their cis stereochemistry and NOE between $\mathrm{H} 12$ and H9e protons and between NH13 and H9a, H7' protons were consistent with the R,R-stereochemistry of C10, C12 centers (see NOE's and the conformation for that fragment of the molecule in the scheme above). (Copies of spectrum are available upon request.) 